\title{
Multifunctional lipidic nanocarriers for effective therapy of glioblastoma: recent advances in stimuli-responsive, receptor and subcellular targeted approaches
}

\author{
Manasa Manjunath Hegde ${ }^{1}$. Suma Prabhu ${ }^{2}$. Srinivas Mutalik ${ }^{3} \cdot$ Abhishek Chatterjee $^{4}$. Jayant S. Goda ${ }^{4}$ \\ B. S. Satish $\operatorname{Rao}^{5}$
}

Received: 23 June 2021 / Accepted: 18 October 2021 / Published online: 8 November 2021

(c) The Author(s) 2021

\begin{abstract}
Background Glioblastoma, or glioblastoma multiforme (GBM), remains a fatal cancer type despite the remarkable progress in understanding the genesis and propagation of the tumor. Current treatment modalities, comprising mainly of surgery followed by adjuvant chemoradiation, are insufficient for improving patients' survival owing to existing hurdles, including the blood-brain barrier (BBB). In contemporary practice, the prospect of long-term survival or cure continues to be a challenge for patients suffering from GBM. This review provides an insight into the drug delivery strategies and the significant efforts made in lipid-based nanoplatform research to circumvent the challenges in optimal drug delivery in GBM.

Area covered Owing to the unique properties of lipid-based nanoplatforms and advancements in clinical translation, this article describes the application of various stimuli-responsive lipid nanocarriers and tumor subcellular organelle-targeted therapy to give an idea about the strategies that can be applied to enhance site-specific drug delivery for GBM. Furthermore, active targeting of drugs via surface-modified lipid-based nanostructures and recent findings in alternative therapeutic platforms such as gene therapy, immunotherapy, and multimodal therapy have also been overviewed.

Expert opinion Lipid-based nanoparticles stand out among the other nanocarriers explored for GBM drug delivery, as they support both passive and active drug targeting by crossing/bypassing the BBB at the same time minimizing toxicity and projects better pharmacological parameters. Although these nanocarriers could be a plausible choice for treating GBM, indepth research is essential to advance neuro-oncology research and enhance outcomes in patients with brain tumors.
\end{abstract}

Keywords Glioblastoma multiforme $\cdot$ Blood-brain barrier $\cdot$ Lipid-based nanocarriers $\cdot$ Lipid-polymer hybrid nanoparticles $\cdot$ Targeted drug delivery

B. S. Satish Rao

rao.satish@manipal.edu

1 Department of Radiation Biology \& Toxicology, Manipal School of Life Sciences, Manipal Academy of Higher Education, Manipal, Karnataka 576104, India

2 Fusion Pharmaceuticals Inc, 270 Longwood Rd. S., Hamilton, ON L8P 0A6, Canada

3 Department of Pharmaceutics, Manipal College of Pharmaceutical Sciences, Manipal Academy of Higher Education, Manipal, Karnataka 576104, India

4 Department of Radiation Oncology, Tata Memorial Centre, Homi Bhabha National Institute, Dr. E Borges Road, Parel, Mumbai, Maharashtra 400012, India

5 Directorate of Research, Manipal Academy of Higher Education, Manipal 576104, Karnataka, India

\section{Introduction}

Glioblastoma, also referred to as glioblastoma multiforme (GBM), is a grade IV astrocytoma and is one of the most devastating forms of cancer. Based on the tumor location in the brain, brain tumors have either focal or generalized symptoms, such as irregular headaches associated with aphasia and seizures (DeAngelis 2001). First-line of treatment for GBM involves surgical resection of the tumor accompanied by radiotherapy and chemotherapy. The current gold-standard drug for glioma is temozolomide (TMZ), an alkylating agent, and its effect is limited due to the resistance caused by O6-methylguanine-DNA methyltransferase (MGMT). Moreover, TMZ has a short half-life, due to which it has to be administered at high doses and for a prolonged duration, which result in a series 
of side effects including thrombocytopenia, neutropenia, and lymphopenia (Chamberlain 2010). Although multimodality therapy comprising of surgery, radiotherapy and TMZ has improved survival outcomes (Stupp et al. 2010), the results are nowhere comparable to those achieved in most of the other advanced-stage solid tumors. This poor outcome can be attributed to the challenges in surgical resection, and GBM's resistance to conventional chemotherapeutics because of several factors including presence of efflux pumps on the blood-brain barrier (BBB), which causes drug efflux, leading to poor drug delivery to tumor sites. Hence, novel targeted treatment approaches to combat these obstacles are of utmost priority. In this regard, nanomedicine, a vast branch of nanotechnology, has great potential for overcoming most of the therapeutic barriers and also effectively bypassing the BBB. Nanoparticles (NPs) of appropriate shape, size, physiological stability, and the ability to incorporate bioactive molecules may facilitate increased solubility and can successfully bypass the BBB. In literature, a large spectrum of nanocarriers such as metal-based ones, for example, gold and silver NPs; iron oxide NPs; polymeric NPs; and lipid-based NPs have been explored and are reported as promising drug delivery vehicles for GBM (Johnsen et al. 2017; Norouzi 2020; Norouzi et al. 2020; Pottoo et al. 2020). However, despite the various advantages, many of these nanocarriers innately face drug delivery-related issues such as normal tissue toxicity, non-specific delivery, and the risk of unintended drug release (Mishra et al. 2019; Li et al. 2021). Among all the nanocarriers explored for treating GBM, lipid-based NPs are preferred owing to the biocompatibility of the products used, such as lipids, triglycerides, fatty acids, and waxes; enhanced penetrability due to desired particle sizes varying between 50 and $300 \mathrm{~nm}$; higher stability due to the advent of a combination of emulsifiers; and increased permeability (Aparicio-Blanco and TorresSuarez 2015; Karim et al. 2016; Barkat et al. 2020). In addition, these nanocarriers can transport bioactive compounds such as nucleic acids and enzymes to target the tumor site. Moreover, they can shield their cargo from degradation while improving its pharmacological effect. Lipid-based nanocarriers, in general, reduce the drug's toxic profile and enhance targeted tissue specificity (Puri et al. 2009; Iqbal et al. 2012). In this review, we emphasize the suitability of liposomes, solid lipid nanoparticles (SLNs), and nanostructured lipid carriers (NLCs)-the three major types of lipid nano formulations that have demonstrated controlled and predictable release of drug load at the tumor site and have promising prognostic outcomes in terms of GBM tumor progression. Further, properties of the tumor microenvironment (such as acidic $\mathrm{pH}$, mildly elevated temperature, and hypoxia) have also been explored to distribute payloads in glioma tumor tissues using stimuli-triggered lipid-based nanocarrier drug delivery systems (DDSs).

\section{Glioma: challenges in clinical management}

The major limiting factor for the design of novel therapeutics that are effective for the brain is the BBB. The brain parenchyma cells are guarded by the BBB, which majorly consists of endothelial cells, pericytes, and astrocytes. The brain microvascular endothelial cells are linked by tight junctions (TJs), which form a barrier for most of the drugs entering the brain. This structure poises a major challenge to transport therapeutic molecules into the brain if they are not surface functionalized. Further, many active efflux transporters present on the BBB restrict the entry of the drug to the brain (Ballabh et al. 2004; Abbott et al. 2006; van Tellingen et al. 2015). In GBM, the blood-brain tumor barrier (BBTB) resides between tumor tissues and blood capillary vessels. As tumor grows, BBTB loses its integrity, thereby becoming leakier and more dysfunctional than the BBB. Owing to the increased permeability and retention effect, BBTB becomes more prone to transporting nanocarriers and enables drug accumulation at the tumor site (Karim et al. 2016; Arvanitis et al. 2020). Hence, the structural and functional integrity of BBB/BBTB must be considered for the development of effective targeting molecules for gliomas. The mechanism of drug resistance includes the upregulation of drug efflux transporters, which can ooze out the drug from the cells (Ds 2015; Mason 2015; Li and Sahi 2016). Efflux transporter in the BBB acts as a barrier in the drug delivery process, where it actively pumps out a variety of drugs such as anti-cancer agents via the cell membrane (Gulsun et al. 2017; Sriraman et al. 2017; Tellingen et al. 2018). Glioma stem cells (GSCs) can self-renew, initiate a tumor, and differentiate from reconstituting the initial tumor mass. GSCs are involved in tumorigenicity and are resistant to chemotherapy and radiation therapy (Bao et al. 2006; Eramo et al. 2006; Chen et al. 2012). It has been reported that GSCs express high levels of vascular endothelial growth factor (VEGF) and stromal-derived factor-1 (SDF-1)—crucial factors involved in angiogenesis that contribute to tumor growth (Bao et al. 2006; Folkins et al. 2009; Huang et al. 2010). They are often commonly present in hypoxic environments, as they serve as essential components in neo-angiogenesis. Moreover, GSCs stimulate tumor-associated endothelial cell proliferation, expansion, and tube formation through intercellular signaling, resulting in tumor vascularization (Filatova et al. 2013). Thus, GSCs could be a potential target for the development of multitargeted therapeutics for better prognosis. Besides, hypoxia is another significant problem in GBM, as cells in hypoxic conditions are susceptible to migrate into healthy brain tissue, resulting in tumor invasion, which is 
the leading cause of death in patients suffering from glioma. Hypoxia-inducible factors (HIFs), specifically HIF2 $\alpha$, are the major molecular markers expressed in hypoxic tumor cells. In response to hypoxia, cells undergo multiple transcription modifications, which significantly impact cellular activities (Soeda et al. 2009). Therefore, HIF2 $\alpha$ could potentially be used to target GSCs in malignant GBM. The presence of hypoxic regions within GBM and other solid tumors is one of the main causes of radio resistance. Consequently, oxygen-mimicking compounds have been studied as effective radiosensitizers in many cancers (Rey et al. 2017). Researchers have put in a lot of effort to improve the limitations in glioma radiotherapy, and nano-radiosensitizers have emerged as a promising treatment choice. Using nanotechnology to develop novel nano-sized platforms that cross the BBB and deliver a high concentration of the drugs into the tumor, thereby increasing tumor response to radiotherapy, is fast becoming an attractive approach to improve radiotherapy outcomes in these tumors (Liu et al. 2017a; Xie et al. 2021).

\section{Current drug delivery strategies for brain tumors}

Although the BBB is essential for the normal functioning of the brain, it becomes a major barrier during therapeutic drug interventions. Further, even though several novel methods to circumvent the BBB are being created, most of these therapeutic approaches are associated with inevitable side effects (Aryal et al. 2014; Barua et al. 2014). The various strategies being applied to breach the barrier can be classified into invasive and non-invasive approaches. The strategies or treatment modalities that are being extensively employed for the betterment of treatment outcomes are indicated in Fig. 1.

\section{Targeting glioblastoma multiforme: an effective approach via nanocarriers}

Nanocarriers are effective DDSs that have recently gained a lot of attention as a potential platform for targeted drug delivery, including the brain. This is been explored not only as a multifunctional platform for efficient drug delivery but also as diagnostic tools. It must be noted that for the successful transport of the drug/biomolecules to the brain, these carriers should possess required physicochemical properties
Fig. 1 Current drug delivery strategies for glioblastoma multiforme treatment based on breaching the blood-brain barrier

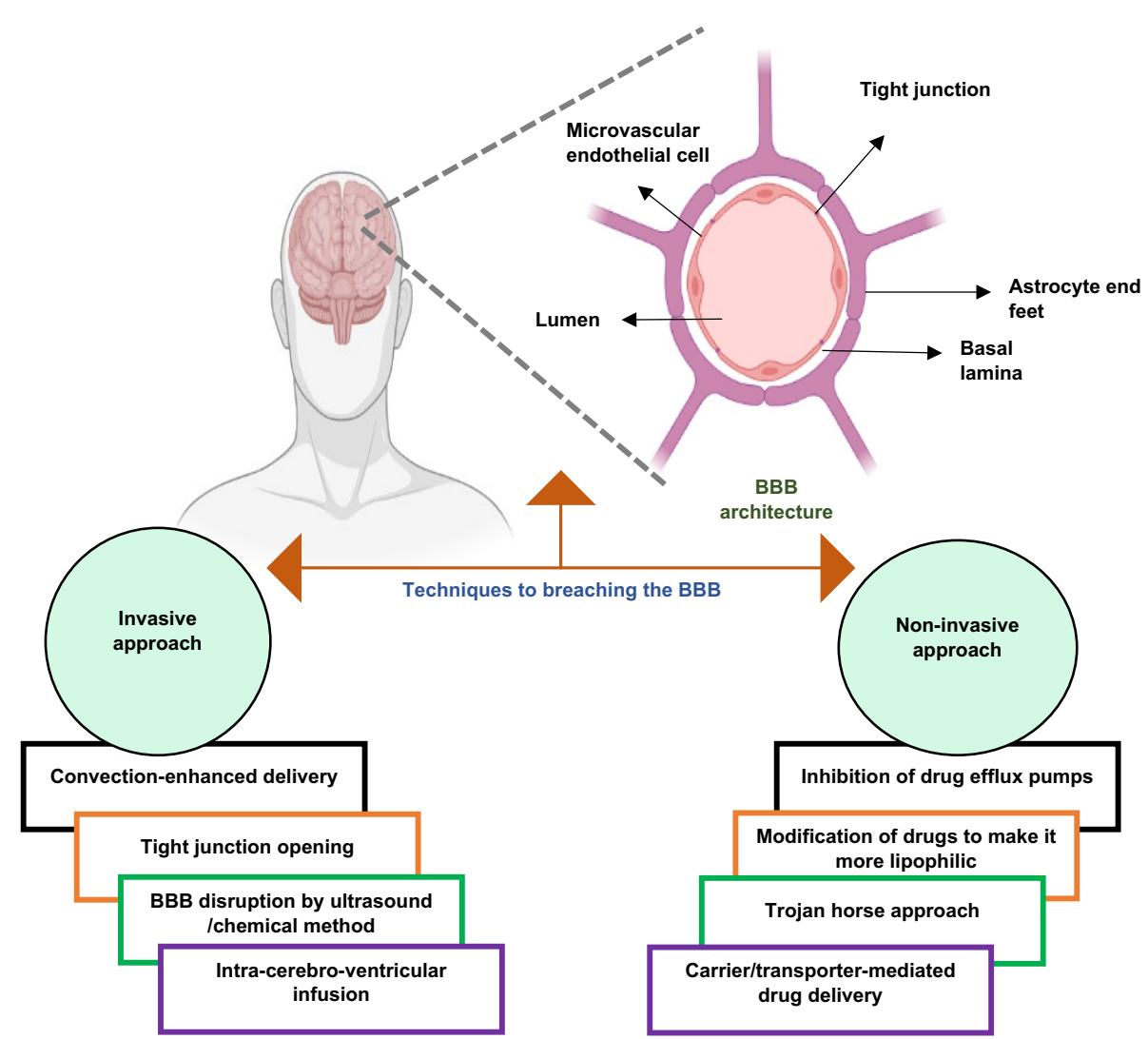


such as low molecular mass, lipid solubility, and less number of hydrogen bonds to cross the BBB (Banks 2016; Warren 2018).

Nanocarriers could be structured by varying their composition and, consequently, tailored to achieve site-specific drug delivery to GBM (Bhaskar et al. 2010; Lombardo et al. 2019). In recent years, NPs such as liposomes, dendrimers, micelles, and organic, inorganic, metallic, and polymeric nanocarriers have extensively been used as drug delivery vehicles for site-specific treatments, as shown in Fig. 2 (Bennet and Kim 2014; Zhu and Liao 2015; Din et al. 2017). For efficient drug delivery into the tumor, the nanocarriers should (1) carry the drugs to the targeted site via endocytosis, (2) have a size $<200 \mathrm{~nm}$ and not be immunogenic, (3) escape from opsonization and clearance by the reticuloendothelial system (RES), (4) be able to guard the drug against any mode of degradation and have long-plasma circulation time, and (5) should recognize intra/extracellular environmental stimuli and increase the cellular internalization. A comparative account of widely used three different lipidbased nanocarriers are described in Fig. 3. Many studies show that particle size and surface charge, as well as the presence of a targeting moiety on the surface, are important factors in brain targeting. However, nanocarriers are cleared by the RES consisting of phagocytic cells (monocytes and macrophages) and may preferentially attract phagocytes, which may enhance the rate of plasma clearance (He et al. 2010). On the other hand, surface-charged nanocarriers do not aggregate easily, which can increase their shelf-life. Therefore, surface charge decides the fate of the nanocarriers. However, to target the brain, cationic nanocarriers are more useful, as they can easily pass through the BBB by adsorptive-mediated transcytosis (AMT; Yang et al. 2017).

\section{Lipid-based nanocarriers: flexible platform for targeted drug delivery to the brain}

Lipid-based or lipidic nanocarriers are extensively used DDSs, as they offer versatile properties such as high drug loading efficiency, low toxicity, biocompatibility, sustainedrelease behavior, protection against drug degradation, stability, and suitability for drug delivery via various routes. Lipid-based NPs such as liposomes, NLCs, SLNs are considered vital nanocarrier systems for brain targeting (Mansor et al. 2019; Niu et al. 2019). Moreover, to overcome the glitches associated with polymer NPs, lipid-based nanocarriers have gained more attention for effective targeting (Shukla et al. 2018; Harshita et al. 2020). However, despite the advantages of using nanocarriers in various diseases, including GBM, they can still potentiate various toxicities induced by oxidative stress, inflammation, and DNA damage

Fig. 2 Various nanoparticles explored as drug delivery systems for glioblastoma multiforme

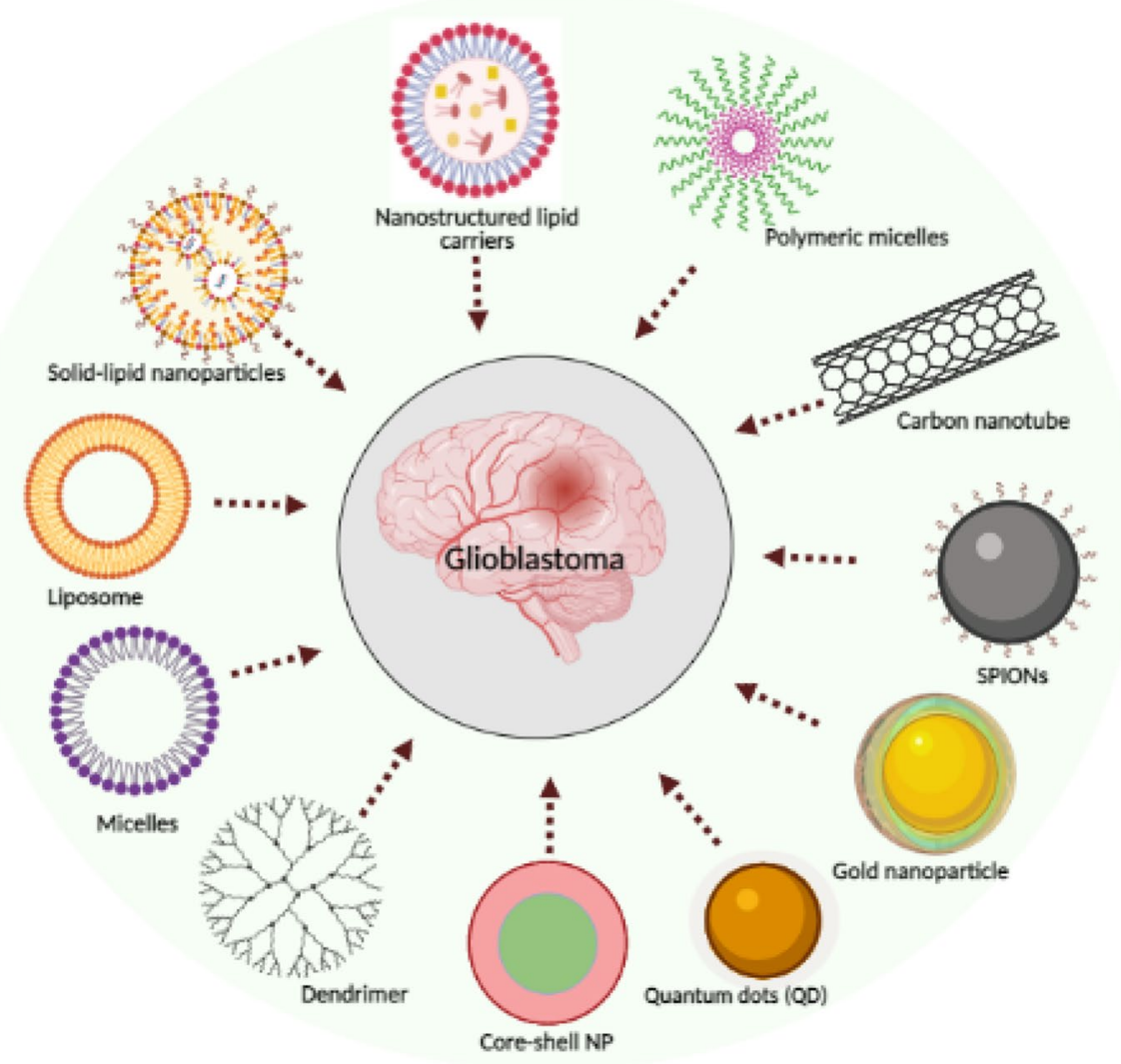


Fig. 3 Comparative account of lipid-based nanocarriers. Adapted from Poonia et al. (2016) and Ghasemiyeh and Mohammadi-Samani (2018)
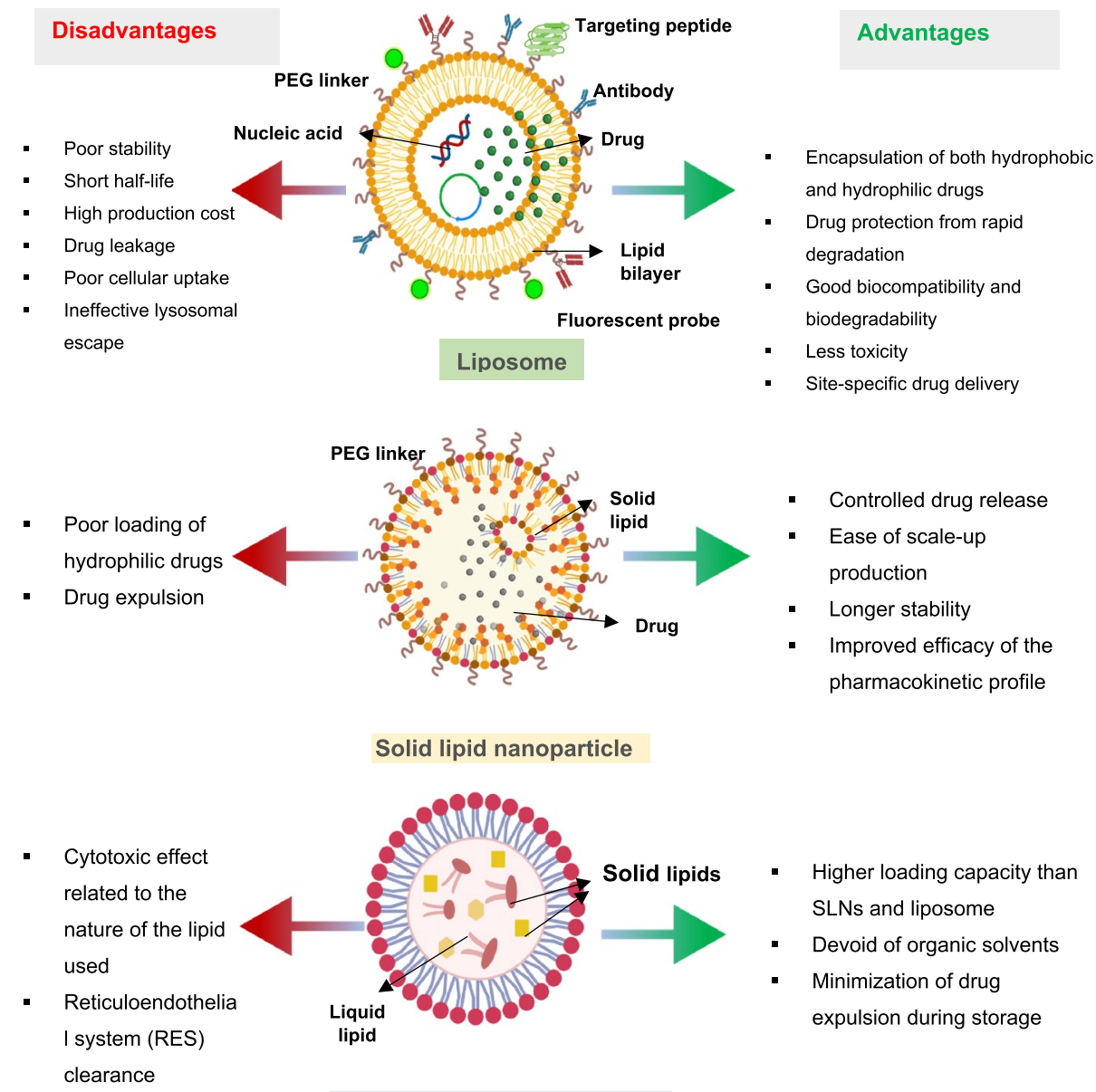

Nanostructured lipid carriers to healthy cells (Bahadar et al. 2016). Studies have revealed that phagocytes' particle size, diameter, and uptake are crucial factors that affect the disease site (Riaz et al. 2018; Liao et al. 2019). Based on the physicochemical properties and the method of preparation, lipid-based nanocarriers can be classified into different categories: (1) liposomes, spherical vesicles having a lipid bilayer made up of phospholipids; (2) niosomes, consisting of cholesterol and non-ionic surfactants; (3) transferosomes, which are similar to liposomes and are composed of stabilized lipid matrix; (4) SLNs, which are made up of a solid lipid core; and (5) NLCs, comprising liquid lipid core surrounded by a solid lipid layer (Tapeinos et al. 2017). Some of the important lipids used in the formulation of lipid nanocarriers are listed in Table 1.

\section{Transport mechanism of lipidic nanocarriers across the blood-brain barrier}

Anticancer agent-loaded lipid-based nanocarriers are known to be able to cross the BBB. Hence, endogenous drug transport pathways provide a promising avenue for research into bypassing drug payloads across the BBB. Larger and/or hydrophilic molecules such as lipoproteins, transferrin, and insulin use various receptors that are highly expressed on the luminal side of endothelial cells. These receptors allow substances to pass through the BBB via endocytosis and transcytosis. There are three major classes of transport pathways for BBB-targeted lipid-based nanocarriers: (1) AMT, (2) receptor-mediated endocytosis (RME), and (3) transportermediated transcytosis (TMT) (Khan et al. 2018). AMT refers to the delivery of drugs using nanoparticulate systems functionalized with cell-penetrating peptides or cationic proteins via electrostatic interactions (Hervé et al. 2008). Solutes, such as glucose or amino acids, bind to a protein transporter on one side of the membrane, allowing the protein to change conformation; this results in the transfer of the substance from a high to a low concentration on the other side of the membrane. Glucose transporters (GLUT) are proteins that help get glucose from the bloodstream to the brain; these have received a lot of interest in the study of BBB-targeted lipidic nanocarriers (Qin et al. 2010). RME transports drugloaded lipid nanocarriers across the BBB endothelium lining through highly selective and unique receptors with natural/ 


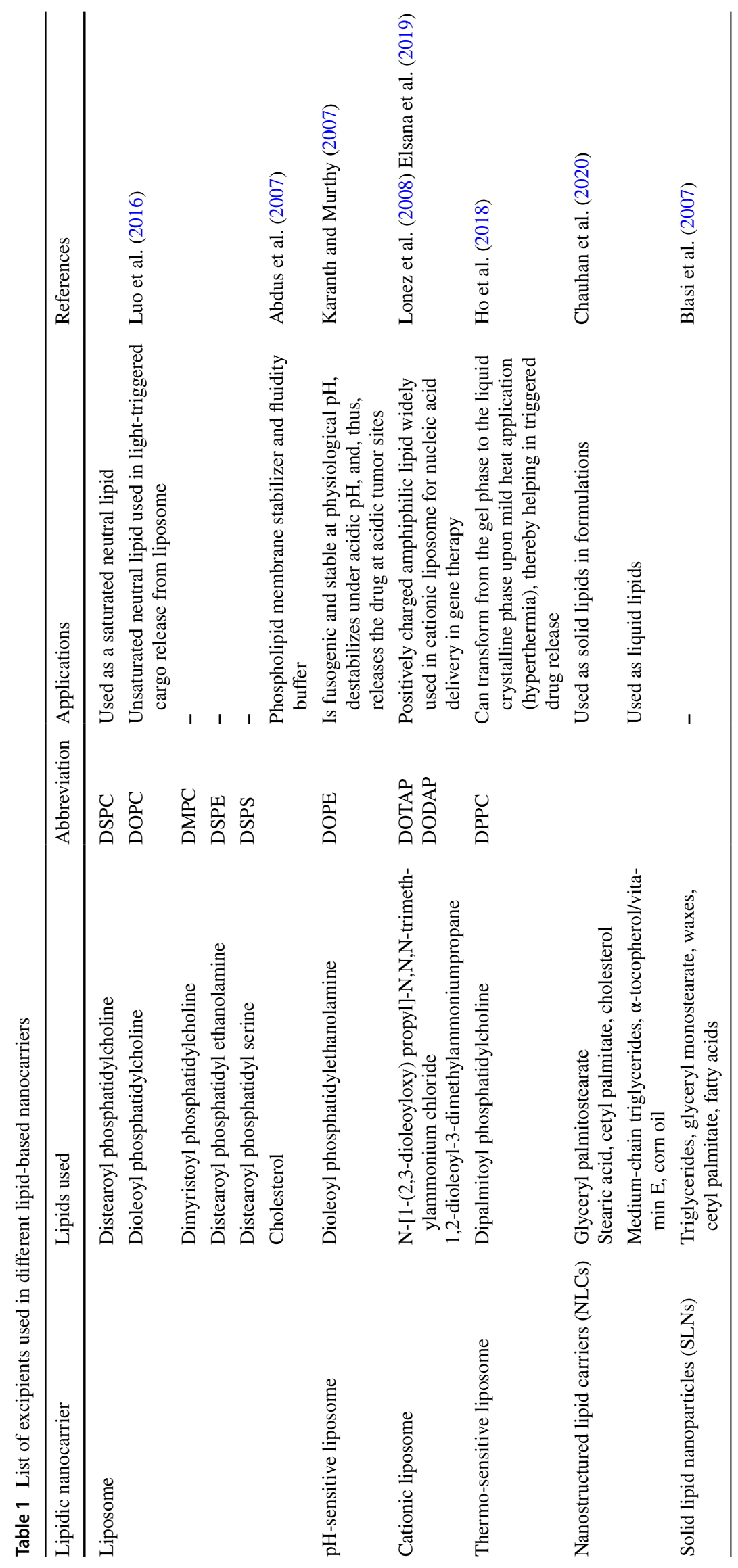


artificial (antibody) ligands and releases them into the brain (Rip et al. 2009).

\section{Liposomes}

Liposomes are considered first-generation lipid-based NPs, which were one of the first-ever drug delivery carriers used to transport anticancer and antimicrobial drugs into cells (Yingchoncharoen et al. 2016; Shukla et al. 2018). Liposomes resemble the cell membrane that can deliver several hydrophobic as well as hydrophilic pharmaceuticals without altering their basic properties. These pharmaceuticals include proteins, peptides, small molecules, RNAs, etc. Owing to their versatility and many advantages over the other NPs, recent research has focused on formulating the multitargeted liposomes for central nervous system-related diseases, specifically for brain tumors. One of the advantages that liposomes possess is that they can cross the BBB via receptor-mediated transcytosis (RMT) or AMT (Liu and Lu 2012; Liao et al. 2019) and, therefore, can be used to treat various types of brain tumors. Once they cross the BBB/ BBTB, liposomes preferentially accumulate at the tumor site; thus, non-specific targeting and consequent side effects of the entrapped therapeutics can be drastically reduced, which, in turn, improves the drug safety profile (Koukourakis et al. 2000). Liposomes that are delivered systemically should retain their physicochemical properties to cross the BBB/BBTB effectively. They should be small $(<200 \mathrm{~nm})$, have adequate plasma circulation time, and be bound to ligands that are internalized by cerebroendothelial cells on their surface (Alyautdin et al. 2014). The BBB passage of liposomes can be increased by making them functionalized with specific ligands such as transferrin, lactoferrin, and peptides (Zheng et al. 2015; Hayward et al. 2016). Several preclinical studies show that grafting endogenous ligands or monoclonal antibodies onto the liposome surface improves GBM targeted drug delivery as compared to a passively targeted nanocarrier. Active targeting of multifunctional liposomes is a promising approach for GBM-targeted drug delivery. Owing to their easy and large-scale manufacturing capability, tunable structure, ability to cross the BBB, and preferential aggregation within tumor tissue, they act as an appealing DDS for the treatment of GBM. The applications of liposome as a therapeutic moiety and in theragnostic for GBM are discussed subsequently.

\section{Solid lipid nanoparticles}

SLNs are new-generation lipid-based nanocarriers that have been developed as a substitute for liposomes and other polymeric NPs (Müller et al. 2000). Problems associated with other nanocarriers, such as systemic toxicity, rapid drug clearance, and poor loading capacities, led scientists to focus on SLNs, as they combine the advantages of conventional drug carriers. Therefore, extensive studies have been carried out on SLNs and their applications in numerous fields (Khames et al. 2019). These NPs can be fabricated by using lipids such as stearic acid, acetyl palmitate, and stearyl alcohol, which are commonly used to prepare other lipidic nanocarriers. Surfactants, including polysorbate 80, polyvinyl alcohol, and sodium cholate, are also being used to stabilize SLNs (Sarangi and Padhi 2016). The morphology of SLNs is spherical, with sizes ranging from 40 to $1000 \mathrm{~nm}$. As the name indicates, the lipid component is present in a solid state at both body and ambient temperature (Blasi et al. 2007b). The appropriate selection of lipids and surfactants and their ratio could affect their physicochemical characters, such as particle size, stability, and drug release behavior. Compared to other carrier systems, SLNs has numerous benefits, including low cost with high-scale production, reliability, and excellent drug release profile. Moreover, low water solubility allows controlled and sustained release of the encapsulated drug. Additionally, their longevity allows them to be used for a prolonged period, which may be useful for treating brain tumors for which sustained drug release with high concentrations of the drug within the tumor is essential. Furthermore, SLNs are biocompatible and can be sterilized readily, and their fabrication techniques do not involve the use of organic solvents, which could otherwise unfavorably affect the finished product's toxicity (Pardeshi et al. 2012). In this regard, three models of drug incorporation in SLNs have been reported based on the production method: (1) core-shell model, which consists of a drug-enriched outer shell surrounding the lipid core; (2) solid solution model, in which drug is dispersed in a lipid matrix with strong drug lipid interaction; and (3) outer-shell model, with a drugenriched core (Üner and Yener 2007).

\section{Nanostructured lipid carriers (NLC)}

NLC emerged as second-generation lipid NPs to overcome the limitations posed by the first-generation carrier systems, i.e., SLNs and liposomes. They are made up of a solid lipid matrix containing a mixture of solid and liquid lipids and surfactants in an aqueous phase, which enhances the stability and drug loading capacity (Pardeike et al. 2009). They can be classified based on the incorporated drug moiety location in an NLC. The first type is NLC type I, also called an imperfect crystal model, consisting of a disordered solid lipid matrix with void spaces for more drug accumulations. The second is the multiple-type NLC, characterized by high liquid lipid content, which is more useful for loading lipophilic drugs. The amorphous model is the third type of 
NLC, in which a lipid matrix is present in the homogenous amorphous state (Selvamuthukumar and Velmurugan 2012). The basic component of an NLC that affects stability, drug loading, and sustained release is the lipid component. Commonly used solid lipids are diglycerides, steroids, fatty acids, and waxes, as they are designated as "generally recognized as safe" (GRAS) (Shah et al. 2015). Further, oleic acid, olive oil, propylene glycol dicaprylocaprate are some of the liquid lipids (oils) used in making NLCs.

\section{Lipid-drug conjugates (LDC)}

LDC are lipidic prodrugs in which drug molecules are either covalently or non-covalently linked to lipids such as fatty acids and glyceraldehydes. LDCs are prepared by utilizing the advantage of lipid metabolic pathways, which may target organs (Lambert 2000). LDCs have multiple advantages, including enhanced drug loading and oral bioavailability. Various conjugation methods are utilized to synthesize LDCs depending upon chemical structures and the interaction of lipids and drugs. Cholesterol and cholic acid are steroids that have been widely used to conjugate drug molecules through the hydroxyl group present on the steroid ring. As cancer cells overexpress low-density lipoprotein receptors (LDLRs) and require large amounts of cholesterol for rapid growth, many studies have led to the synthesis of cholesterol-conjugated anticancer drugs to target tumor cells (Radwan and Alanazi 2014). LDCs can improve BBB penetration by increasing lipophilicity of the drug or target receptors, which facilitates lipid transportation to the brain by crossing the BBB (Nikanjam et al. 2007).

\section{Stimuli-responsive lipidic nanocarriers}

Liposomes have been used as drug delivery vehicles for many years, as they possess numerous advantages over conventional DDSs. However, poor bioavailability of the encapsulated drug, instability in humans, and their uncontrolled release have limited their applications in the medical field (Akbarzadeh et al. 2013; Sercombe et al. 2015). To overcome these problems, DDSs that can respond to different external and internal stimuli for controlled drug release are highly desirable. Stimuli-responsive DDSs target a specific site for their drug delivery in a controlled manner, resulting in an adequate concentration of drug reaching the target site with reduced side effects. Based on various stimuli approaches, different types of nanocarriers can be synthesized; these have been classified as endogenous stimuli-controlled and exogenous stimulicontrolled systems. In the endogenous stimuli-controlled system, drug release is controlled by various enzymes, temperature, $\mathrm{pH}$, etc.; conversely, in the exogenous stimuli-controlled system, external stimuli such as radiation, magnetic field, focused ultrasound are used to regulate drug release from the nanocarriers (Fig. 4; Gopalan et al. 2020). Liposomes with stimuli-responsive ability constitute a promising method to enhance therapeutic efficacy. Stimuli-responsive liposomes can be prepared by using phase transition lipids and stimuli-responsive chemical moieties into liposomes that respond to stimuli (Kono 2001; Yuba 2020). Triggering the drug release from lipidbased nanocarriers by applying external stimuli facilitates site-specific delivery (Fleige et al. 2012; Mura et al. 2013; Lee and Thompson 2017).
Fig. 4 Schematic representation of stimuli-responsive lipid nanocarriers for glioblastoma multiforme
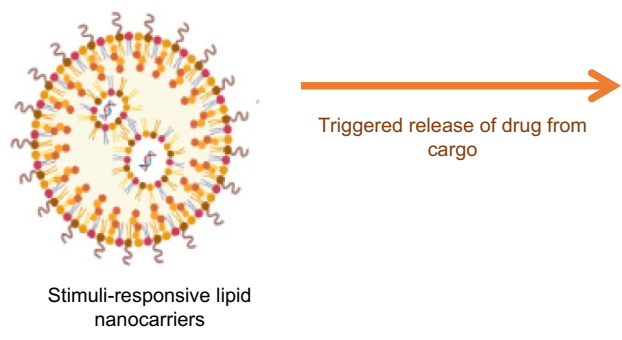
release of drug at tumor site 


\section{Thermo-sensitive lipidic nanocarriers}

Temperature influences the metabolic activity of cells. The human body maintains a physiological temperature of $37{ }^{\circ} \mathrm{C}$ for intracellular reactions, and when the body temperature exceeds the limit of $43-45^{\circ} \mathrm{C}$, hyperthermia sets in. In such conditions, cells cannot maintain their regular metabolism due to the denaturation of proteins and degradation in the cellular enzymatic activity. The increased temperature over physiological levels or "hyperthermia" can impose irreversible damage to the tumor cells through microtubule disruption, inhibition of DNA synthesis, inhibition of DNA synthesis and repair, and changes in receptor expression (Aoki et al. 2004). In the case of brain tumors, the application of mild hyperthermia (characterized by a few degrees $\left(4-5{ }^{\circ} \mathrm{C}\right)$ above the physiological temperature) has been used along with radiotherapy and chemotherapy as a non-invasive approach to boost the efficacy of treatment (Pacheco-Torres et al. 2015). Therefore, one of the most studied stimuli-responsive drug delivery mechanisms for cancer treatment is thermoresponsive drug delivery. Thermo-sensitive liposomes are made up of lipids that can alter their physical properties in response to temperature changes, thereby causing drug release. To produce thermo-sensitive liposomes, dipalmitoyl phosphatidylcholine (DPPC) and 1,2-distearoyl-snglycero-3-phosphocholine (DSPC) are commonly used. As lipids have a transition temperature $\left(\mathrm{T}_{\mathrm{m}}\right)$ of $41-43{ }^{\circ} \mathrm{C}$, the permeability of the lipid membrane is enhanced at this temperature, resulting in enhanced drug release from the liposomes (Yatvin et al. 1978). For instance, a similar temperature-responsive liposomal formulation ThermoDox ${ }^{\mathrm{TM}}$ is currently in phase III clinical trials for the treatment of hepatocellular carcinoma (Ho et al. 2018). Thermo-sensitive liposomes (TSL-Dox) are made by using lipids such as DPPC, 1,2-distearoyl-sn-glycero-3-phosphoethanolamine-N-[methoxy(polyethylene glycerol)-2000] (DSPE$\mathrm{PEG}_{2000}$ ), and mono stearoyl phosphatidylcholine (MSPC) encapsulated with doxorubicin (Dox). The formulation was evaluated for localized release upon hyperthermia applications in the canine model. TSL-Dox showed higher tumor cellular uptake, confirming its ability to cross the BBB. This formulation achieved delivery of therapeutic doses with sustained release up to 15-30 min of hyperthermia treatment at the tumor site as compared with unheated tissue (Bredlau et al. 2018). In another study, a novel thermo-sensitive liposome was developed using cellpenetrating peptides (CPPs), anti-glioma antibodies, and super paramagnetic iron oxide nanoparticles (SPIONs). In this study, the authors used GBM-specific cell-penetrating peptide (P1NS) and an antibody (Tenascin-C) to direct the functionalized liposome to target glioma cells without being taken out by other endothelial cells. Additionally, co-encapsulating Dox and SPIONs inside the liposome exhibited thermo-triggered drug release under an alternating magnetic field (AMF). The results demonstrated enhanced drug release with reduced cytotoxicity of Dox to healthy brain tissues, compared to the non-functionalized liposome. Incorporation of SPIONs allowed triggered Dox release in a temperature-dependent manner due to heat generation by external AMF. Therefore, dual-functionalized liposomes with thermo-sensitivity have emerged as a promising drug delivery approach for GBM treatment (Shi et al. 2019). In a recent study, a new type of dual sensitive SLN has been synthesized and its efficacy tested against various cancers, including glioma (Świętek et al. 2020). As it was incorporated with iron oxide NPs, this nanocarrier had magnetic and temperature sensitivity, and it demonstrated heat dissipation when an external magnetic field was applied. The SLNs showed dose-dependent cytotoxicity against drug-resistant cells such as glioma U251 cells as well as other cells such as Jurkat and HL-60 leukemia cell lines even without any drug conjugation (Świętek et al. 2020).

\section{Photo-thermal lipidic nanocarriers for glioblastoma multiforme: an evolving technology}

Photodynamic therapy (PDT) is an evolving technique that uses non-ionizing excitation of light and a photosensitizing agent to induce an antitumor immune response (Dolmans et al. 2003). PDT is known to produce heat and singlet oxygen species and affect vascular endothelial cells, resulting in tumor necrosis, apoptosis, and tissue ischemia (Krammer 2001; Castano et al. 2005). This treatment has been employed for many types of cancer, such as skin cancer and non-small cell lung cancer, as well as in treating malignant gliomas (Akimoto 2016; Shafirstein et al. 2016). When PDT is applied for brain tumors, the use of safe excitation light is necessary to avoid collateral damage to the neighboring neural cells (Dolmans et al. 2003). PDT employs non-toxic dyes as photosensitizers (PSs), which are activated by absorbing light at wavelengths between 400 and $800 \mathrm{~nm}$, resulting in an excited triplet state. An excited triplet state can create reactive oxygen species (ROS) by photochemical reactions in the presence of oxygen, which can kill cancer cells. However, most of the PSs are hydrophobic molecules with a high tendency to aggregate, lack tumor selectivity, and undergo rapid clearance by the kidneys (Licha et al. 2000; Saxena et al. 2003). Hence, the encapsulation of PSs could enhance their performance. A few NPs such as titanium oxide, fullerenes, and some types of quantum dots themselves act as PSs; therefore, these can be utilized in PDT (Li 2013; Lucky 
et al. 2015). In a study, indocyanine green (ICG), a potent PS for PDT, was incorporated with 1,2-dioleoyl-snglycero3-phosphoethanolamine (DOPE) in liposomes in the rat 9L GBM model for effective PDT (Shibata et al. 2019). When ICG-liposomal formulation was intravenously administered to the tumor-bearing animals, the accumulation of NPs in the tumor site was notably increased, which was thereafter activated by near-infrared (NIR) light. Magnetic resonance imaging (MRI) images of the brain showed significant tumor growth suppression in NIR-irradiated animals. Interestingly, brain histopathological analysis revealed the accumulation of CD8+T cells and macrophages in tumor cells with a marked expression of heat shock protein-70 (HSP70), which was not activated by mild hyperthermia alone $\left(45^{\circ} \mathrm{C}\right)$ and IL-2-mediated immune reaction. Remarkably, the effectiveness of this treatment was not seen in nude rats. These findings showed that NIR irradiation in combination with a novel NP (ICG and liposome combination) could effectively elicit an immune reaction by inducing HSP70 expression, which can lead to various immunological chain reactions, facilitating tumor cell death (Shibata et al. 2019).

\section{pH-sensitive lipidic nanocarriers}

$\mathrm{pH}$-sensitive liposomes have numerous applications in different areas of drug delivery, as they can be tuned to attain the $\mathrm{pH}$ required for the human physiological state. The unique feature of being sensitive to external $\mathrm{pH}$ makes these nanocarriers one of the ideal choices for drug formulation in cancer therapy. However, liposome use in $\mathrm{pH}$-triggered drug delivery is limited due to the absence of sensory components. The incorporation of $\mathrm{pH}$-sensitive lipids helps in sensing mild $\mathrm{pH}$ differences between the healthy tissues and tumor microenvironments, which further triggers drug release at the tumor site (Karanth and Murthy 2007).

Gliomas, especially GBM, have a low $\mathrm{pH}$, ranging from 5.9 to 6.8 , while a healthy brain tissue has a $\mathrm{pH}$ of 7.1 (Hjelmeland et al. 2011). A functionalized stealth liposome engineered with an ion channel as a sensory component that could sense the small $\mathrm{pH}$ change $(0.2 \mathrm{pH}$ unit precision) in the tumor microenvironment has been developed, which assists in image-guided drug delivery devices for GBM (Pacheco-Torres et al. 2015). This liposomal formulation was made by using DOPC, cholesterol, and DSPE-PEG ${ }_{2000}$, encapsulating Gd-diethylendiaminopentaacetic acid (GdDTPA), a paramagnetic chelate that acts as an MRI contrast agent that can be readily detected in vivo during MRI. Further, the addition of a mechanosensitive channel of large conductance (MscL), a sensory ion channel extracted from Escherichia coli, into the liposome bilayer creates a temporary pore on the liposome when $\mathrm{pH}$ is slightly acidic $\mathrm{pH}$ 6.6-7.0) in C6 glioma tumors of mice. These pores allow leakage of imaging agents at the tumor site, which could be tracked non-invasively via MR spectroscopy. This novel study would enable effective future drug delivery designs for cancer therapy (Pacheco-Torres et al. 2015). In another study, $\mathrm{Li}$ et al. developed a dual-modified $\mathrm{pH}$-responsive liposome to target glioma. Paclitaxel (PTX)-loaded liposomes were co-modified with dNP2, a highly potent cell-penetrating peptide that can transport its cargo to different brain cells, exhibiting superior cellular internalization and tumor microenvironment and $\mathrm{pH}$-cleavable folic acid, a ligand for attacking glioma cells. The addition of acidcleavable folic acid and dNP2 resulted in improved transportation through the BBB and showed increased cellular uptake and cytotoxicity of the loaded drug in C6 glioma cells at pH 6.8. As compared to non-modified liposomes, this $\mathrm{pH}$-sensitive nanocarrier maintained BBB permeability and increased aggregation in orthotopic glioma tumors. This multitargeting technique has been shown to be an effective drug delivery mechanism for the management of glioma ( $\mathrm{Li}$ et al. 2018).

\section{Hypoxia-responsive tumor targeting approach}

Nanotechnology, a growing field, can open a new path in targeting glioma hypoxic zones, as NPs can be modified such that they accumulate in low-pH hypoxic regions and thus release the drug for effective therapy. In a recent study, a hypoxia-responsive ionizable liposome loaded with gene silencing small interference RNA (siRNA) was developed to selectively target the hypoxic tumor site of GBM. A pololike kinase 1 (PLK1) siRNA was used to inhibit PLK1, a highly expressed protein in glioma. Hypoxia-sensitive positively charged lipid-incorporated liposomes were designed in such a way that it could release siRNA at hypoxic conditions and at low extracellular $\mathrm{pH}$ by increasing positive charge on the surface. Significant tumor growth inhibition confirmed the effective targeted delivery of siRNA into the glioma tumor site (Liu et al. 2017c). To improve the radiosensitizing effect on gliomas, angiopep-2-lipid-poly-(metronidazoles) n (ALP-(MIs)n) hypoxic radiosensitizer-polyprodrug NPs were created. The radiotherapy hypoxic sensitization effects of ALP-(MIs)n polyprodrug NPs could deliver hydrophobic chemotherapy, allowing for concurrent chemoradiotherapy while also activating the release of hydrophobic chemotherapeutics under hypoxic conditions. In vitro and in vivo findings indicate that these ALP-(MIs)n polyprodrug NPs can selectively attack gliomas and significantly inhibit their growth (Hua et al. 2018). Like the above study, Liu et al. (2017b) engineered a novel liposome having hypoxic sensitization as a Dox carrier to achieve a synergistic chemo/ radiotherapy treatment of glioma. In both in vitro and in vivo 
studies, MLP/Dox was shown to increase cellular uptake and facilitate Dox release inside hypoxic glioma cells (Liu et al. 2017b). In another study, to produce synergistic results, an angiopep-2 (A2)-modified TMZ-loaded lipid-poly (hypoxic radiosensitized polyprodrug) NP was formulated. This particle exhibited several unique features such as a hydrophobic core wherein TMZ could be encapsulated; nitro groups of the hydrophobic core, which are transformed into hydrophilic amino groups under low-oxygen conditions to imitate the oxygen environment and enhanced sensitization to radiotherapy; and at the core-shell lipid monolayer interface to modify the angiopep-2, a ligand for low-density lipoprotein receptor-related protein-1 (LRP-1), which increases tumor specificity (Zong et al. 2019).

\section{Receptor-mediated drug delivery}

Cancer cells overexpress several receptors as compared to normal cells (Zeromski 2002). Therefore, targeting the overexpressed receptors could enhance cellular uptake and accumulation of anticancer therapeutics, as depicted in Fig. 5. The receptor-mediated DDS has been widely studied in various fields, including GBM. Some receptors have been reported to be highly expressed on both the BBB and gliomas, which provide targeting sites for receptor-mediated drug delivery (Wang et al. 2015). A receptor-mediated DDS consists of nanocarriers that are conjugated with various ligands targeting different receptors such as transferrin receptors (TfRs), lactoferrin receptors (LfRs), epidermal growth factor receptors (EGFRs), and folate receptors (FRs; Cui et al. 2013; Liu et al. 2013; Su et al. 2014). Targeting the receptors could facilitate both transportation of drugs across the BBB as well as their internalization into glioma cells. TfR is essential in cellular iron uptake, which regulates the amount of iron delivered to the cells (Calzolari et al. 2010). Across the globe, researchers have utilized transferrin to transfer the drugs across the BBB. TfR 1 and TfR 2 are overexpressed on cerebral endothelial cells and many other cells of malignant gliomas (Voth et al. 2015; Prabhu et al. 2017). Lactoferrin is another abundantly expressed receptor in the neurons and brain endothelial cells. A list of receptors explored to target gliomas using liposomes is summarized in Table 2.

\section{Subcellular targeted therapy for GBM}

Biological macromolecules such as DNA, siRNAs, and antibodies specifically target the cell interior. They target organelles such as mitochondria, endoplasmic reticulum (ER), nucleus, and other subcellular structures. To facilitate the drug or macromolecule accumulation at the intracellular site, these macromolecules need to translocate through the lipophilic cell membrane. However, most of the drugs do not reach the targeted subcellular organelle unless they are actively transported (Rajendran et al. 2010). To escape from the endocytic pathway, intracellular drug delivery can be achieved by using surface-modified nanocarriers. Intracellular targeting ligands or moieties such as $\mathrm{pH}$-responsive polymer, antimicrobial peptide (AMP), CPP, mitochondrial localization signal (MLS), and nuclear localization signal (NLS) (Jhaveri and Torchilin 2016) are being incorporated with nanocarriers to target specific organelles for effective
Fig. 5 Schematic representation of receptor-mediated drug delivery using lipid nanocarriers. Functionalized nanoparticles conjugated with receptorspecific ligand/antibody will be internalized by the cells, thereby targeting the nucleus, which leads to apoptosis

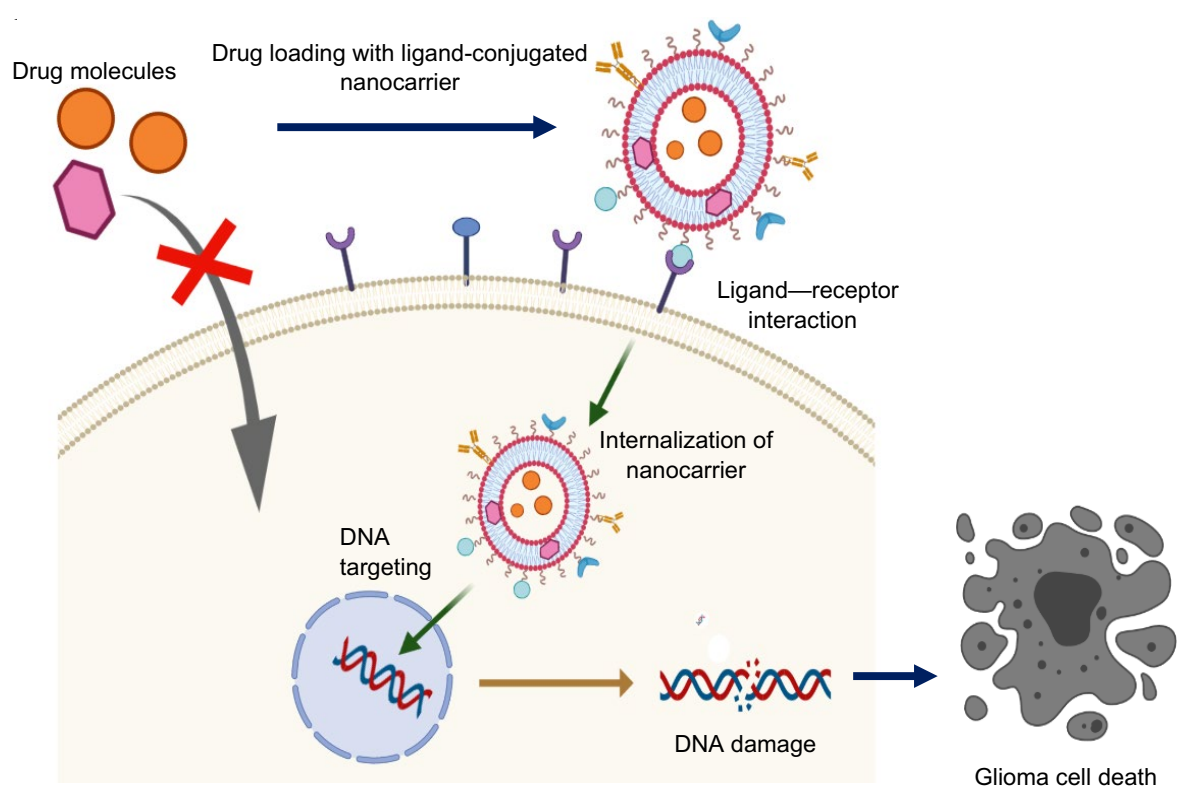




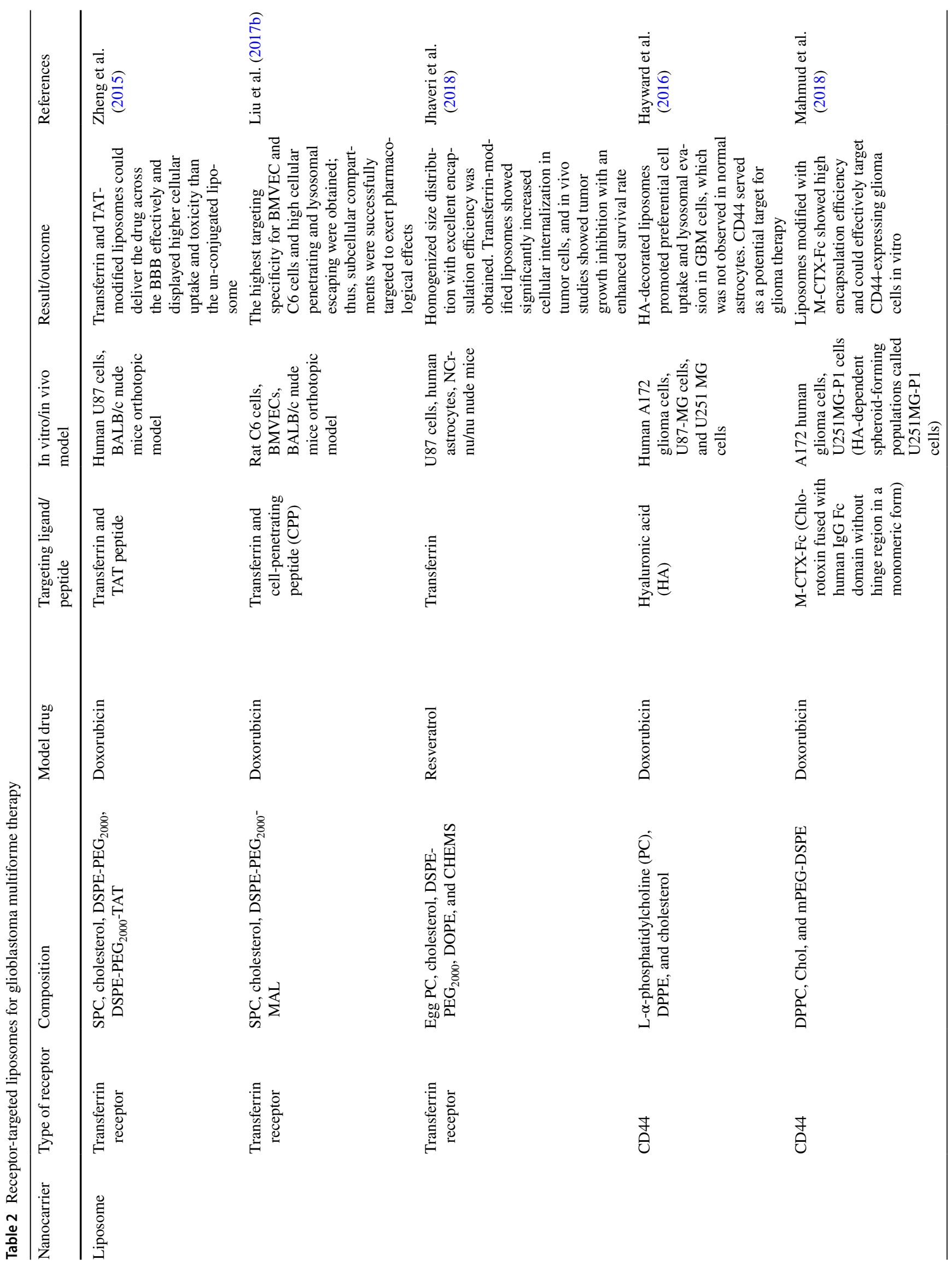




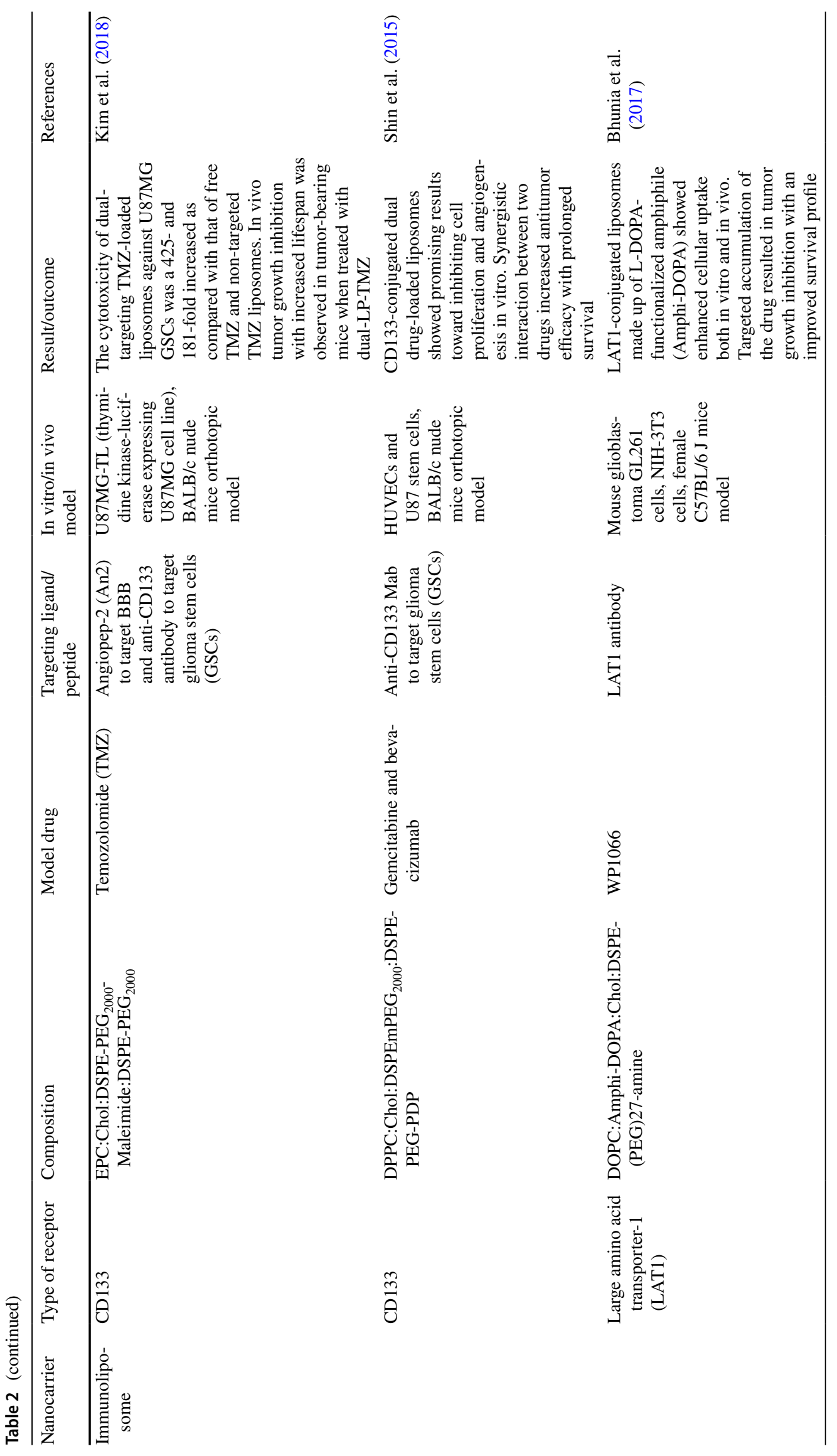




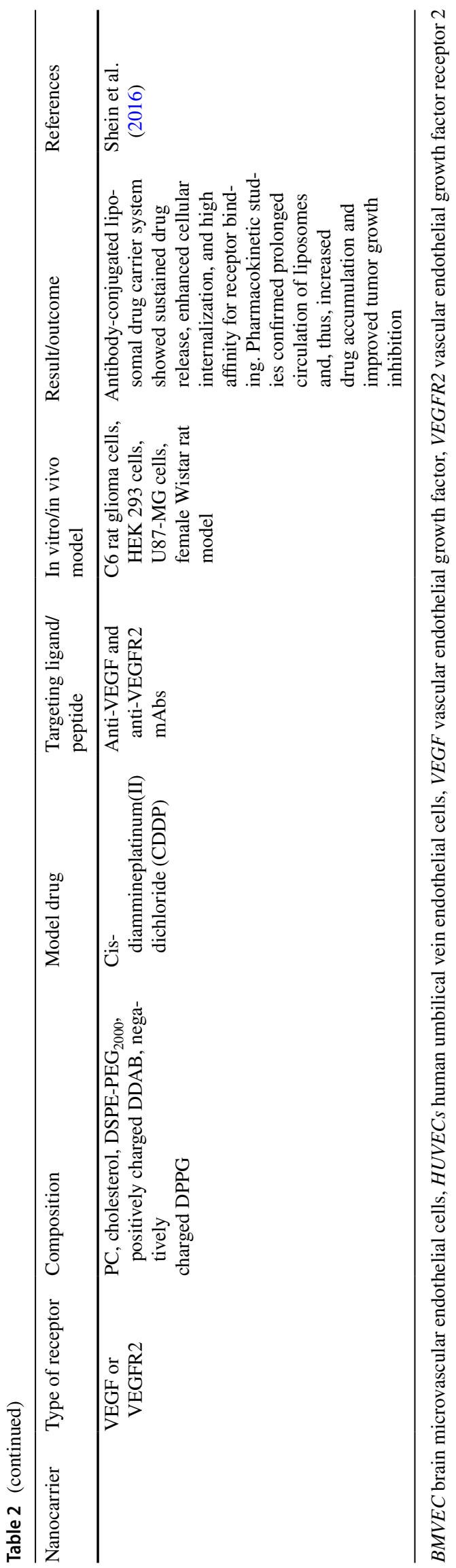


drug delivery to treat various diseases, including GBM (Parodi et al. 2015). Thus, subcellular targeting via nanocarriers could increase the potential of various drug therapies by reducing the quantity of payloads.

\section{Mitochondrial and Golgi/endoplasmic reticulum targeting lipidic nanocarriers}

Malignant tumors, including glioma, generate adenosine triphosphate (ATP) through aerobic glycolysis, which may result in mitochondrial dysfunction. This impairment could be associated with abnormalities in mitochondria-dependent apoptotic pathways. Mitochondria play a vital role in cellular proliferation and apoptosis; therefore, mutations in apoptotic genes like Bcl-2, p53 may lead to inherent chemoresistance in GBM (Costantini et al. 2000; Ordys et al. 2010; Battogtokh et al. 2018). Certain clinically approved drugs such as ceremide, PTX, and CD-437 are known to directly target mitochondria to trigger apoptosis. However, to date, no drug can target mitochondria in GBM. In this context, structural abnormalities and mitochondrial dysfunctions in malignant gliomas and their implications are poorly understood. Moreover, the application of lipid-based nanoplatforms to target subcellular organelle is still emerging. Liposomes functionalized with a lipid-glucose derivative loaded with daunorubicin to target GSC mitochondria have been shown to accumulate in mitochondria by crossing the BBB, thus exhibiting a cell-killing effect by inducing apoptotic signaling pathways in mitochondria as well as glioma cells (Zhao et al. 2016a). Quercetin (QUE) nanoliposomes are unique in that they target tumor cells to induce type III (necrotic) programmed cellular death, which is independent of apoptosis and caspase activity. Wang et al. (2012) assessed QUE nanoliposome-treated C6 glioma cells for necrosis, ROS production level, and loss of mitochondrial membrane potential by flow cytometry. Further, lactate dehydrogenase (LDH) activity, as well as cytochrome $\mathrm{C}$ release, was evaluated to confirm necrotic cell death. The findings showed that nanoliposomes induce necrotic cell death in glioma cells and thus provide the base for developing novel therapeutic approaches in this domain. Golgi complex/bodies are cell organelles that, along with the ER, form a part of the secretory pathway. It has been shown that the secretory pathway can sense the stress stimuli in cells and thus initiate cell death signaling (Momoi 2004). In GBM, ER and Golgi complex contents are in a higher proliferative phase than the normal adult glial cells (Silvestre et al. 2009). Hence, targeting the Golgi-ER network could provide a platform for better therapeutic opportunities in the field of targeted drug delivery. Moreover, actively targeting intracellular organelles helps deliver the drug accurately to the specific intracellular site of the tumor cell. The interleukin 13 (IL-13) peptide-conjugated nanocarrier, loaded with docetaxel (DTX) and designed for intracellular delivery to target GBM, was observed to localize primarily to the Golgi apparatus and mitochondria, indicating their involvement in intracellular trafficking and sorting of NPs (Gao et al. 2014). Results suggested that accumulated NPs in the Golgi apparatus may cause toxicity that could affect protein modification and exertion, which further leads to the apoptosis of glioma cells (Gao et al. 2014).

\section{Lipidic nanocarrier-based combinatorial drug approach for glioblastoma multiforme}

The presence of $\mathrm{BBB}$, tumor heterogeneity, multidrug resistance (MDR), poor pharmacokinetics of the chemotherapeutics limited the magnitude of benefit derived from monochemotherapy in GBM. To overcome these challenges, drug combinations with different working mechanisms have elicited considerable interest recently (Zhao et al. 2020). However, the enhanced efficacy of drug combinations comes at the cost of increased toxicity. To achieve a therapeutic drug level, high doses are given, which leads to significant off-target toxicity. For example, a well-known drug TMZ is administered in high doses $\left(200 \mathrm{mg} / \mathrm{m}^{2} /\right.$ day $)$ to treat glioma patients, resulting in side effects such as neutropenia, lymphopenia, and thrombocytopenia (Perry et al. 2017). Hence, combining different drugs with a reduced dose of each drug would be a better choice in treating GBM. However, cytotoxic drug combinations failed to address the problems associated with drug distribution and poor cellular uptake at the desired tumor site. Different drug combination approaches have been reached in clinical trials to treat GBM. Some of them showed better results when treated in combination than in single-drug therapy (Davis et al. 2008; Alam et al. 2010; $\mathrm{Lu}$ et al. 2014). Often, effective preclinical drug-combination strategies failed to show their efficacy in clinical trials due to multiple reasons such as differences in individual drug distribution, metabolism, and excretion. These failures need to be addressed by alternative drug delivery strategies. With evolving technology, different therapeutic approaches have been applied to mitigate non-targeted drug distribution within the brain. Out of all the strategies, nanotechnologybased targeted drug delivery approaches show a promising outcome with enhanced chemotherapeutic efficacy (Zhao et al. 2020). Encapsulating multiple drugs in a single nanocarrier ensures delivery at the appropriate intracranial site in desirable concentrations. Lipid-based NPs have been widely explored in the drug combination delivery field. Among them, liposomes are best suited for transporting multiple chemotherapeutic agents, as they can carry hydrophilic drugs in an aqueous core and hydrophobic drugs in the lipid bilayer. A combination of drug-loaded NPs along with other treatment modalities such as radiation therapy has displayed 
remarkable outcomes in pre-clinical studies. PEGylated liposomal Dox known as Caelyx ${ }^{\circledR}$ combined with radiotherapy resulted in higher Dox accumulation in brain tumor than in normal brain tissues in GBM patients (Koukourakis et al. 2000). In a recent study, hydrogels consisting of prodrug lauroyl-gemcitabine $\left(\mathrm{GemC}_{12}\right)$, lipid nanocapsules (LNCs), and lipophilic PTX were developed for a combined local delivery approach for GBM (Bastiancich et al. 2019). The addition of $\mathrm{GemC}_{12}$, which is located at the oil-water interphase of LNC, led to the spontaneous formation of hydrogels, which was unaffected by the presence of PTX. In in vitro studies, cells showed combined and synergistic efficacy on GL261 GBM cells (Bastiancich et al. 2019). In addition, SLNs and LNC have gained attention in the area of lipid-based delivery systems.

\section{Surface-modified lipidic nanocarriers for GBM}

\section{Modification with chlorotoxin}

Chlorotoxin (ClTx) is a powerful toxin exploited for tumor targeting. It is a 36-amino acid peptide derived from the venom of scorpion Leiurus quinquestriatus. It is known to bind to chloride channel-3 (ClC-3) and matrix metalloproteinase 2 (MMP2), which are upregulated in GBM but not in normal brain tissues (Soroceanu et al. 1998; Deshane et al. 2003). When this toxin binds to the chloride channel present on glioma cells, it blocks the influx and efflux of chloride ions, with consequent adverse effects on cellular homeostasis. However, very few studies have reported the application of CITx as a targeting moiety to deliver the drugs or other diagnostic agents (Kievit et al. 2010; Huang et al. 2011). Results of studies on U87MG cells (expressing both MMP-2 and ClC-3) demonstrated the increased targeting ability of liposome with CITx conjugation via MMP-2 dependent uptake both in in vitro and in vivo studies. A similar study was done using ClTx as a targeting ligand conjugated with Dox-loaded liposomes for glioma therapy and imaging. Here, the targeting compound was synthesized by conjugating CITx to DSPE-PEG ${ }_{2000}$-NHS and further added with lipids to make liposomes. Formulated liposomes confirmed enhanced cellular uptake through RME due to the presence of CITx. In contrast, unconjugated liposomes showed poor cellular internalization. In vivo studies corroborated with in vitro studies; accumulation of CITx-Dox liposome increased at the brain tumor site than at any other part of the body. However, drug distribution observed in the brain and toxicity of CITx-liposomes on normal brain endothelial cells remain a matter of concern (Xiang et al. 2011). Although ClTx is reported as non-toxic to mammals (Shen et al. 2005), detailed studies on cellular and tissue toxicity need to be done to validate its potential as an anticancer drug delivery vehicle. Thus, CITx can be beneficial in several ways to target the tumor, as it has the potential to target specific receptors in glioma.

\section{Aptamer modification}

Off-target consequences of currently available treatment modalities affect GBM patients' quality of life and outcomes. To overcome the existing hurdles associated with targeted drug delivery, in recent decades, antibody development to target tumor-specific receptors has been made an integral part of the research. However, antibody production is a time-consuming, costly process and high doses need to be administered for effective responses, thereby limiting clinical efficacy of antibodies (Chames et al. 2009). To address the drawbacks of antibody utilization, an alternative approach using aptamers is under investigation (Gan et al. 2017). Aptamers are single-stranded oligonucleotides that can be derived from an in vitro process known as systematic evolution of ligands by exponential enrichment (SELEX). Studies have been taken up to target brain tumors by utilizing aptamers and NPs (Guo et al. 2011; Monaco et al. 2017; Zhao et al. 2019).

\section{Peptide modification}

In recent times, peptides are gaining attention as potent targeting moieties, as they exhibit protein functionalities and possess a high degree of modification at the molecular level. Peptides display many advantages over proteins, including high stability, good specificity, and less susceptibility to eliciting adverse immunogenicity (Sanna et al. 2014). Many studies have shown the effective utilization of artificial peptides in biomedical applications (Raucher 2019). The efficiency of peptide function can be increased by conjugating them with non-biological compounds such as polymers and metallic compounds, which would address the drawbacks of the peptides (Shu et al. 2013). Among all the available conjugation approaches, NPs are an attractive option for improving the functionality of the peptides. Consequently, peptide conjugation with NPs has been a widely applied method in treating GBM (Raucher 2019). Further, CPPs can potentially be used to cross the BBB, as they are small peptides that can easily translocate the membrane with enhanced cellular uptake. Among widely used peptides for brain transport, Wang et al. tested peptide- 22 and cyclic arginine-glycine-aspartic acid (RGD)/c(RGDfK), which are specific ligands for LDLR and integrin $\alpha v \beta 3 / \alpha v \beta 5$, respectively. Dual peptide-conjugated liposomes loaded with Dox displayed good physicochemical characterizations, as they have a particle size below $105 \mathrm{~nm}$ with $>95 \%$ drug entrapment efficiency. In vitro and in vivo studies demonstrated 
that both $\mathrm{c}(\mathrm{RGDfK})$ and peptide-22 conjugation could significantly enhance the cellular uptake in U87 glioma cells as well as navigation across the BBB by increase in the liposome accumulation at the tumor site (Chen et al. 2017). In a similar study, tumor-specific pH-responsive cell-penetrating peptide $\mathrm{H}_{7} \mathrm{~K}\left(\mathrm{R}_{2}\right)_{2}$ was designed, and $\mathrm{H}_{7} \mathrm{~K}\left(\mathrm{R}_{2}\right)_{2}$-modified Dox-loaded liposomes were developed to target GBM (Zhao et al. 2016b). Moreover, SLNs have also been widely used as a carrier for glioma-targeting peptides. Angiopep-2, a small peptide having 19 amino acid residues, specifically binds to LRP-1 and has higher BBB penetrating ability when conjugated with SLNs for the delivery of DTX for anti-glioma therapy (Kadari et al. 2018). In another study, ultra-small NLCs were utilized as dual drug carriers with peptide modification for targeting glioma (Basso et al. 2020). In this study, atorvastatin and curcumin (drugs that exhibit potential anticancer uses) were incorporated in NLCs, which were modified with hyaluronic acid (HA), cRGDfK, and $\mathrm{H}_{7} \mathrm{~K}\left(\mathrm{R}_{2}\right)_{2}$ peptide for effectively inhibiting GBM tumor growth. As RGD is a widely used peptide for targeted drug delivery, NLCs filled with TMZ and functionalized with an RGD peptide were tested in GBM therapy. The nanostructures that had a scale of $120 \mathrm{~nm}$, a positive surface charge of $+28 \mathrm{mV}$, and an encapsulation efficiency of $85 \%$ inhibited the U87MG glioma cell, making them a potentially promising structure for treating GBM (Song et al. 2016). Together, these studies indicated that lipidic nanocarriers embellished with tumor-specific peptides can offer potentially efficacious options in GBM therapy.

\section{Lipid-polymer hybrid nanoparticles}

In the past two decades, polymeric NPs and liposomes have emerged as two dominant classes of nanocarriers in the field of targeted drug delivery due to their high efficiency of drug encapsulation and site-specific delivery (Zhang et al. 2008). However, these NPs are fraught with the problem of short circulation time, drug leakage, and non-specific targeting. To overcome these limitations, a new-generation delivery system has been developed by hybridizing both polymeric NPs and liposomes: lipid-polymer hybrid nanoparticles (LPHNPs). This robust hybrid nanocarrier displayed high encapsulation efficiency, sustained-release kinetics, good serum stability, and sensitivity to triggered release. As its name indicates, LPHNPs combine the characters of both liposomes and polymeric NPs. The structure consists of three layers: The innermost layer is a drug-encapsulating polymeric core, followed by a monolayer of lipid surrounding the core, and the outermost layer is the PEG layer acting as a stabilizer for prolonged systemic circulation of LPHNPs (Mukherjee et al. 2019). The most common polymer used for core preparation is poly(lactic-co-glycolic acid) (PLGA) because of its biocompatibility and excellent biodegradability. Its unique core is highly flexible to modulation so that a variety of drugs can be loaded. Several types of lipids can be used to fabricate the NP outer shell with lecithin, cholesterol, DPPC along with PEG for surface modification (Guo et al. 2011). Studies have focused on treating GBM by modifying the surface with various targeting ligands. In a recent study, lipid-coated polymeric NPs were modified with RGD as a targeting peptide (Shi et al. 2015). The core was made up of PLGA and loaded with DTX. Synthesized nanoformulations displayed good physicochemical characteristics with size $110 \pm 13.5 \mathrm{~nm}$ and $77 \%$ drug entrapment efficiency. A 4.13-fold increased anti-proliferative activity was shown by RGD-NP compared with PLGA-NPs in C6 glioma cells. When the animals were treated with a drug with fluorescent dye-loaded RGD-NPs, the strongest fluorescence signals were observed at the glioma tumor site. Therapy was associated with an increased median survival rate (Shi et al. 2015). Indeed, combination therapy using chemotherapeutics and nucleic acid is always a better option to overcome drug resistance-related problems in cancer. A study adopted this strategy by combining an antifolate agent pemetrexed and anti-microRNA 21 (miR-21) encapsulated in PLGA polymer-based hybrid lipid NPs for the treatment of GBM (Küçüktürkmen et al. 2017). A relatively recent study used farnesyl thiosalicylic acid (FTA), a Ras protein inhibitor, loaded in lipid-polymer hybrid nanocarriers for passive transportation through the BBB due to their relatively smaller size $(164 \pm 10.3 \mathrm{~nm})$ and lipophilic nature (Sekerdag et al. 2017). The synthesized formulations were administered by both intranasal and intravenous routes. The MRI and histopathological results displayed the effectiveness of intranasal administration of FTA-loaded LPHNPs on tumors in treated animals over intravenous application with relatively fewer side effects and systemic accumulation (Sekerdag et al. 2017). Collectively, all these studies provide evidence for successful applications of LPHNPs as a unique mode of treatment for GBM.

\section{Lipid nanoplatform-based alternative therapeutic strategy}

\section{Gene therapy}

Latest efforts on developing more effective strategies to target brain tumors have yielded mixed results. Among all the strategies, gene therapy has attracted huge attention in the context of glioma treatment. The basic concept of gene therapy is the targeted transfer of genetic materials such as microRNAs, siRNA, and aptamers into tumor cells for effective therapeutic purposes (Bansal and Engelhard 2000). However, gene therapy has failed to meet clinical expectations even though it showed promising outcomes 
in preclinical applications. This is due to impediments posed by the brain that act as anatomical barriers and tumor heterogeneity, which hinders vector targeting to the brain (Bansal and Engelhard 2000). NPs are a viable strategy for gene delivery, as they have the potential to target the tumor site (Wang et al. 2016). LPHNPs have been explored as a gene delivery vehicle for drug-resistant GBM. In a study by Yang et al. (2021), LPHNPs were loaded with the CRISPR/ Cas9 plasmid for targeting the MGMT, the gene responsible for TMZ drug resistance. The nanocarrier was conjugated further with cRGD peptide for tumor targeting. The results showed that the nanocarriers could effectively deliver the CRISPR/Cas9 plasmid into the cells, which downregulated the MGMT expression, thus increasing the TMZ sensitivity, as shown in Fig. 6 (Yang et al. 2021).

\section{Immunotherapy}

The CNS has long been called an immune-privileged system due to the lack of a conventional lymphatic system and the difficulties associated with initiating a disruptive T-cell response from the parenchyma. The brain has a noncanonical lymphatic system and is protected from the BBB. Antigens and other molecules that circulate in the brain form a network of meningeal lymphatic vasculature (glymphatic system), which is necessary for the immune response to begin (Sahebjam et al. 2017). GBM possesses tumor-infiltrating lymphocytes, which have been implicated in contributing to poor tumor prognosis (Carpentier and Meng 2006). While treating GBM via immunotherapy, targeting the tumor-immune system activates the host's immune cells/ lymphocytes to destroy GBM cells. Among all the various immunotherapeutic strategies, the nanomedicine-based immunotherapeutic model can be applied to deliver drug moieties with improved pharmacokinetics. Precise and more effective nanocarriers can be designed by modifying them with different immunomodulatory agents such as immune checkpoint inhibitors, modulators of nucleic acids, and other adjuvants (Lou et al. 2019; Hanif et al. 2020). In this context, Zhang et al. (2018) developed the iRGD-liposome, composed of egg phosphatidylcholine, cholesterol, and DSPEPEG-malamide and loaded with immunomodulatory agents PI3K inhibitor and alpha-GalCer agonist of therapeutic T cells, to remove protumor cell populations and trigger the essential antitumor effector cells (chimeric antigen receptor [CAR] T cells). The results obtained from this study showed the anti-tumor efficacy of liposomes capped with immunomodulatory agents with reduced systemic toxicity. The overall survival rate was increased in a genetically engineered immunotherapy-resistant glioma mouse model as compared to CAR T-cell therapy (Zhang et al. 2018). Myeloid-derived suppressor cells (MDSCs) and tumorassociated macrophages (TAMs) play a major role in the induction of immune tolerance in cancer patients. To target these immunosuppressive cells, a recent study described the development of LNCs of size $100 \mathrm{~nm}$ that were able to eliminate both macrophages and tumor cells in GBM patients, thereby opening a new path for exploiting lipid-based nanosystems in the framework of immunotherapy (Pinton et al. 2020).

\section{Nose-to-brain delivery (intranasal delivery)}

The anatomical relationship between the nasal cavity and the CNS, the obvious advantages of nasal delivery of drugs and their distribution into the brain pose a unique challenge in nanomedicine. The drug transport mechanism from the nose to the brain involves different pathways such as the olfactory and trigeminal pathways. Olfactory field neurons and axons terminate in the olfactory bulb, which has close interactions with the CNS due to the presence of olfactory receptors. The active moieties can be absorbed directly into the brain by olfactory and trigeminal receptors in the olfactory region (Erdő et al. 2018; Illum 2000). Today, an increasing number of therapeutics in the market are using the intranasal delivery to make use of these benefits for entering the CNS. NPs improve drug delivery from the nose to the brain by (1) binding with the mucus layer and then injecting a loaded drug into the mucus cells, (2) crossing the mucus layer to be picked up by neurons and get transported to the brain, or (3) crossing the mucus layer to be picked up by neurons and then translocating in nerve axons to enter the brain and release the drug. Mucoadhesive compounds such as chitosan, HA, or low-molecular-weight pectin may be used to coat LNP surfaces to extend olfactory residence time (Kumar et al. 2017). SLNs and NLCs have been described as superior candidates for GBM targeting through the nasal route due to their high biocompatibility, low toxicity, and ease of surface functionalization. Curcumin-loaded NLCs were developed for intranasal delivery to the CNS. An ex vivo study was performed using the nasal cavity of sheep to test carrier nasal permeation, and the permeability coefficient was found to be $3.8 \mathrm{~cm}^{2} / \mathrm{min}$ (Madane and Mahajan 2016). After nasal administration of Cur-NLCs, a biodistribution analysis in rats revealed a substantial increase in curcumin in the brain, with a $\mathrm{C}_{\max }$ of $86.2 \pm 8.1 \mathrm{mg} / \mathrm{g}$ and $\mathrm{T}_{\max }$ of $120 \mathrm{~min}$ (Madane and Mahajan 2016). Intranasal application of TMZ-NLCs has also been developed to target GBM. In vivo experiments have shown that TMZ-NLCs accumulate in the brain more effectively than free TMZ, demonstrating the effectiveness of intranasal NLC administration (Khan et al. 2016). Further, Gartziandia et al. developed achitosan-coated NLC formulations having mucoadhesive properties with positively charged NPs, which enhanced intranasal drug delivery to the brain. The formulations had a long survival period in the nasal epithelium, indicating that they could be used as a 
Fig. 6 Combination therapy for drug-resistant glioma using ultrasound and lipid-polymer hybrid nanoparticles loaded with temozolomide (TMZ) drug and O6-methylguanine-DNA methyltransferase (MGMT) targeting CRISPER/Cas9 plasmid

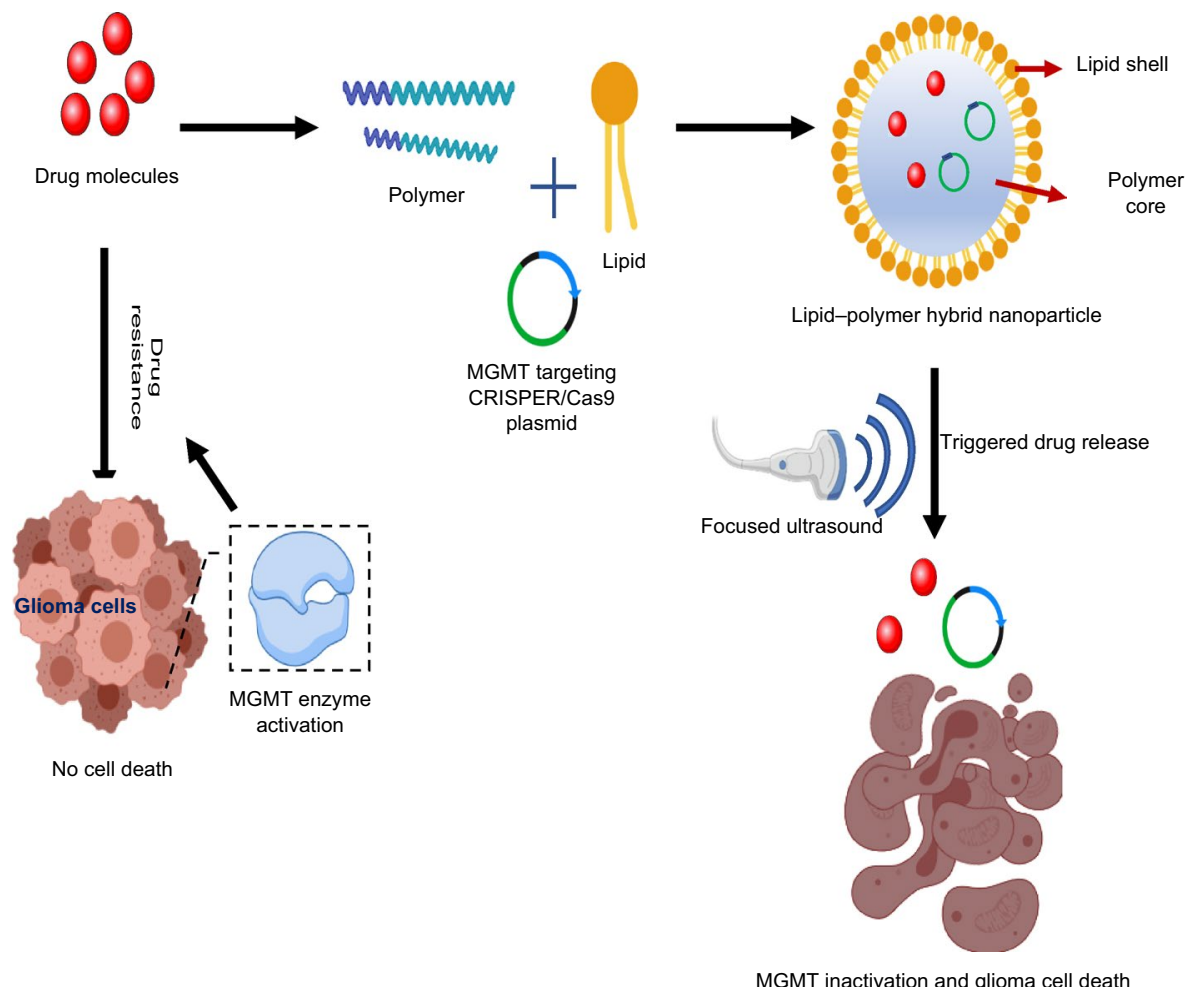

transporter to minimize drug dosage and frequency. However, in vivo fluorescence findings showed that these NPs collected less in the brain than in the lungs, necessitating more modifications to increase its concentration in the brain after being delivered through the nose (Gartziandia et al. 2015).

\section{Multimodal therapy}

The heterogeneity of GBM and its microenvironment have driven researchers to design a variety of treatment approaches. In this context, as opposed to single treatments, combination therapies have raised fresh concerns. For a combination therapy, the selection of a treatment approach is the first and most important consideration; it involves the collection of combination strategies using multiple chemotherapeutic agents as well as the synergy of these techniques. The second major consideration is the therapeutic agentbased delivery mechanism. In a recent study, a multicellular complex model that mimics the microenvironment of a tumor was used to thoroughly characterize and test hybrid magnetic lipid NPs, wherein a physical method (hyperthermia) and a chemical approach utilizing TMS's antitumor effects on 3D models of GBM were investigated (Marino et al. 2019). Further, the heating of lipid magnetic nanovectors, release of chemotherapy drugs, and hyperthermiadependent loss of plasma membrane integrity triggered by the alternating magnetic fields were used to activate lipid magnetic nanovectors. Alternating magnetic fields, lipid superparamagnetic nanovectors, and TMZ were tested independently and in combination on GBM spheroids to see whether the TMZ-loaded lipid superparamagnetic nanovectors had a synergistic impact. The results demonstrated that the combined effect of magnetic field and TMZ on the disintegration of glioma tumor spheroids led to tumor cell death (Marino et al. 2019). Additionally, a hypoxia-responsive lipid-polymer NP for combined fluorescence-guided surgery, chemotherapy, PDT, and photothermal therapy (PTT) was used to create multimodal treatments for glioma $(\mathrm{Xu}$ et al. 2020). In this study, lipid-polymer NP (Dox + ICG)targeting, hypoxia-responsive NPs were used to deliver ICG and Dox, enabling fluorescence-guided surgery and multimodal treatment dependent on the eradication of remaining glioma cells in a novel glioma surgery technique (Xu et al. 2020). The promising results obtained from the pre-clinical studies have been a driving force to conduct clinical trials with lipid-based nanocarriers, specifically with liposome as a drug delivery vehicle for GBM therapy (Table 3).

\section{Future perspective and conclusion}

GBM, the most common and fatal brain tumor, ranks among the least curable cancers owing to its heterogeneity, high proliferating capacity, and intrinsic resistance to radiation and chemotherapy. Existing management methods are often 
Table 3 List of lipid-based nanocarriers under clinical trials for glioblastoma multiforme

\begin{tabular}{|c|c|c|c|c|c|c|c|}
\hline Clinical trial ID & Nanocarrier & Drug used & Study phase & $\begin{array}{l}\text { Study duration } \\
\text { (years) }\end{array}$ & $\begin{array}{l}\text { Number } \\
\text { of patients } \\
\text { (n) }\end{array}$ & Clinical setting & Status \\
\hline NCT 01044966 & Liposome & $\begin{array}{l}\text { Intraventricular } \\
\text { Ara-C+temo- } \\
\text { zolomide }\end{array}$ & $\mathrm{I} / \mathrm{II}$ & 2010-2019 & 12 & $\begin{array}{l}\text { Recurrent glio- } \\
\text { mas }\end{array}$ & $\begin{array}{l}\text { Terminated due to } \\
\text { inadequate patient } \\
\text { enrollment }\end{array}$ \\
\hline NCT 04573140 & $\begin{array}{l}\text { RNA-loaded lipo- } \\
\text { some vaccine }\end{array}$ & $\begin{array}{l}\text { pp65 full-length } \\
\text { lysosome- } \\
\text { associated } \\
\text { membrane } \\
\text { protein (LAMP) } \\
\text { mRNA }\end{array}$ & I & $\begin{array}{l}\text { 2021-2022 (esti- } \\
\text { mated duration) }\end{array}$ & 28 & $\begin{array}{l}\text { Newly diagnosed } \\
\text { pediatric high- } \\
\text { grade gliomas } \\
\text { and adult } \\
\text { glioblastoma } \\
\text { multiforme }\end{array}$ & Not yet recruited \\
\hline NCT 00734682 & Nanoliposome & $\begin{array}{l}\text { CPT-11 (irinote- } \\
\text { can) }\end{array}$ & I & 2008-2014 & 34 & $\begin{array}{r}\text { Recurrent high- } \\
\text { grade gliomas }\end{array}$ & $\begin{array}{l}\text { Completed } \\
\text { The study outcome } \\
\text { measure was to } \\
\text { assess the safety } \\
\text { and pharmacoki- } \\
\text { netics as well } \\
\text { as to determine } \\
\text { the maximum } \\
\text { tolerated dose of } \\
\text { NL CPT-11 in } \\
\text { patients with high } \\
\text { grade recurrent } \\
\text { glioma }\end{array}$ \\
\hline NCT 01906385 & Liposome & - & $\mathrm{I} / \mathrm{II}$ & $2015-2025$ & 55 & $\begin{array}{l}\text { Recurrent glio- } \\
\text { mas }\end{array}$ & $\begin{array}{l}\text { Recruiting process } \\
\text { continued }\end{array}$ \\
\hline NCT 00944801 & $\begin{array}{l}\text { PEGylated lipo- } \\
\text { some }\end{array}$ & $\begin{array}{l}\text { Doxoru- } \\
\text { bicin + temo- } \\
\text { zolomide along } \\
\text { with radio- } \\
\text { therapy }\end{array}$ & $\mathrm{I} / \mathrm{II}$ & 2002-2009 & 63 & $\begin{array}{l}\text { Primary glioblas- } \\
\text { toma multi- } \\
\text { forme }\end{array}$ & $\begin{array}{l}\text { Completed } \\
\text { The study outcome } \\
\text { measure was } \\
\text { to assess the } \\
\text { progression free } \\
\text { survival probabil- } \\
\text { ity at } 12 \text { months } \\
\text { to detect an } \\
\text { improvement of } \\
\text { the PFS-1215.6\% } \\
\text { as compared to } \\
\text { EORTC26981/ } \\
\text { NCIC-CE.3 com- } \\
\text { bination arm }\end{array}$ \\
\hline
\end{tabular}

hindered by non-specific therapies that result in excessive normal tissue toxicity and are characterized by the failure of the therapeutic agents to cross the BBTB and penetrate the tumor microenvironment. Therefore, designing smart and successful therapies for GBM necessitates the use of targeted drug delivery mechanisms that improve drug concentration in tumor tissue while minimizing systemic side effects. Novel investigational therapies should be successful in addressing obstacles such as breaking through the BBB and releasing therapeutic payloads that are not only specific but also effective in the tumor cells, resulting in improving GBM survival and quality of life. Colloidal nanocarriers can be engineered to have a variety of beneficial properties that help in the distribution of therapeutic molecules to brain tumor site and, therefore have piqued the interest of many researchers. Thus, many nanocarriers are now constructed with newly synthesized polymers; however, their toxicity profiles are not well studied. The unpredictability of the influence of different nanocarrier properties on biological fate of nanocarriers and the possibility of long-term toxic effects pose major scientific and regulatory challenges. Owing to these factors, only a small number of lipidic nanocarrier-based DDSs are currently available in the market despite their promising physiochemical characteristics. Consequently, further research is required to develop standardized in vitro, ex vivo and in vivo models and assays that predict the biological and toxicological fate of nanocarriers with greater accuracy. Indeed, nanocarriers such as liposomes, SLNs, lipid-polymer NPs, and LNCs have been shown to enhance drug potency, decrease non-specific 
toxicity, and increase drug stability; with these carriers, biodistribution and drug release kinetics can be precisely regulated, in contrast to conventional formulations. Although concerns about the safety of the raw materials used and regulatory problems persist, the relevance of lipidic nanocarriers as brain-targeted DDSs has been increasing with the number of CNS-related diseases, especially in GBM. Overall, to translate the advances of lipid-based nanomedicine from the bench to the bedside and reap the success of these novel drug delivery platforms, there is an urgent need for a cohesive and amalgamated approach by academia, industry, drug regulatory authorities, and clinicians.

Acknowledgements Authors are thankful to the Director, Manipal School of Life Sciences and MAHE, Manipal for all the support; DST-SERB (EMR/2016/007782) Government of India, New Delhi for providing research support to B. S. Satish Rao and fellowship to Mrs. Manasa Manjunath Hegde. We acknowledge Dr. Nitin K S, postdoctoral researcher, Cape Peninsula University of Technology, South Africa for his editorial assistance. All the images were created with the help of Smart Servier and BioRender.com.

Funding Open access funding provided by Manipal Academy of Higher Education, Manipal.

\section{Declarations}

Conflict of interest All authors (Manasa Manjunath Hegde, Suma Prabhu, Srinivas Mutalik, Abhishek Chatterjee, Jayant S. Goda, B. S. Satish Rao) declare that they have no conflict of interest.

Research involving human and animal rights This article does not contain any studies with human and animal subjects performed by any of the authors of this publication.

Open Access This article is licensed under a Creative Commons Attribution 4.0 International License, which permits use, sharing, adaptation, distribution and reproduction in any medium or format, as long as you give appropriate credit to the original author(s) and the source, provide a link to the Creative Commons licence, and indicate if changes were made. The images or other third party material in this article are included in the article's Creative Commons licence, unless indicated otherwise in a credit line to the material. If material is not included in the article's Creative Commons licence and your intended use is not permitted by statutory regulation or exceeds the permitted use, you will need to obtain permission directly from the copyright holder. To view a copy of this licence, visit http://creativecommons.org/licenses/by/4.0/.

\section{References}

Abbott NJ, Rönnbäck L, Hansson E (2006) Astrocyte-endothelial interactions at the blood-brain barrier. Nat Rev Neurosci 7:41-53. https://doi.org/10.1038/nrn1824

Abdus S, Sultana Y, Aqil S (2007) Liposomal drug delivery systems: an update review. Curr Drug Deliv 4:297-305. https://doi.org/ $10.2174 / 156720107782151269$
Akbarzadeh A, Rezaei-Sadabady R, Davaran S et al (2013) Liposome: classification, preparation, and applications. Nanoscale Res Lett 8:102. https://doi.org/10.1186/1556-276X-8-102

Akimoto J (2016) Photodynamic therapy for malignant brain tumors. Neurol Med Chir (tokyo) 56:151-157. https://doi.org/10.2176/ nmc.ra.2015-0296

Alam MI, Beg S, Samad A et al (2010) Strategy for effective brain drug delivery. Eur J Pharm Sci 40:385-403. https://doi.org/10.1016/j. ejps.2010.05.003

Alyautdin R, Khalin I, Nafeeza MI et al (2014) Nanoscale drug delivery systems and the blood-brain barrier. Int J Nanomed 9:795-811. https://doi.org/10.2147/IJN.S52236

Aoki H, Kakinuma K, Morita K et al (2004) Therapeutic efficacy of targeting chemotherapy using local hyperthermia and thermosensitive liposome: evaluation of drug distribution in a rat glioma model. Int J Hyperthermia 20:595-605. https://doi.org/10.1080/ 02656730410001703186

Aparicio Blanco J, Torres-Suarez A (2015) Glioblastoma multiforme and lipid nanocapsules: a review. J Biomed Nanotechnol 11:1283-1311. https://doi.org/10.1166/jbn.2015.2084

Arvanitis CD, Ferraro GB, Jain RK (2020) The blood-brain barrier and blood-tumour barrier in brain tumours and metastases. Nat Rev Cancer 20:26-41. https://doi.org/10.1038/ s41568-019-0205-x

Aryal M, Arvanitis CD, Alexander PM, McDannold N (2014) Ultrasound-mediated blood-brain barrier disruption for targeted drug delivery in the central nervous system. Adv Drug Deliv Rev 72:94-109. https://doi.org/10.1016/j.addr.2014.01.008

Bahadar H, Maqbool F, Niaz K, Abdollahi M (2016) Toxicity of nanoparticles and an overview of current experimental models. Iran Biomed J 20:1-11. https://doi.org/10.7508/ibj.2016. 01.001

Ballabh P, Braun A, Nedergaard M (2004) The blood-brain barrier: an overview: structure, regulation, and clinical implications. Neurobiol Dis 16:1-13. https://doi.org/10.1016/j.nbd.2003.12.016

Banks WA (2016) From blood-brain barrier to blood-brain interface: new opportunities for CNS drug delivery. Nat Rev Drug Discov 15:275-292. https://doi.org/10.1038/nrd.2015.21

Bansal K, Engelhard HH (2000) Gene therapy for brain tumors. Curr Oncol Rep 2:463-472. https://doi.org/10.1007/ s11912-000-0067-z

Bao S, Wu Q, McLendon RE et al (2006) Glioma stem cells promote radioresistance by preferential activation of the DNA damage response. Nature 444:756-760. https://doi.org/10.1038/natur e05236

Barkat MA, Das SS, Pottoo FH, Beg S, Rahman Z (2020) Lipid-based nanosystem as intelligent carriers for versatile drug delivery applications. Curr Pharm Des 26:1167-1180. https://doi.org/ $10.2174 / 1381612826666200206094529$

Barua NU, Gill SS, Love S (2014) Convection-enhanced drug delivery to the brain: therapeutic potential and neuropathological considerations. Brain Pathol 24:117-127. https://doi.org/10.1111/ bpa.12082

Basso J, Mendes M, Silva J et al (2020) Peptide-lipid nanoconstructs act site-specifically towards glioblastoma growth impairment. Eur J Pharm Biopharm 155:177-189. https://doi.org/10.1016/j. ejpb.2020.08.015

Bastiancich C, Bozzato E, Luyten U et al (2019) Drug combination using an injectable nanomedicine hydrogel for glioblastoma treatment. Int J Pharm 559:220-227. https://doi.org/10.1016/j. ijpharm.2019.01.042

Battogtokh G, Choi YS, Kang DS et al (2018) Mitochondria-targeting drug conjugates for cytotoxic, anti-oxidizing and sensing 
purposes: current strategies and future perspectives. Acta Pharm Sin b 8:862-880. https://doi.org/10.1016/j.apsb.2018.05.006

Bennet D, Kim S (2014) Polymer nanoparticles for smart drug delivery. Appl Nanotechnol Drug Deliv. https://doi.org/10.5772/58422

Bhaskar S, Tian F, Stoeger T et al (2010) Multifunctional nanocarriers for diagnostics, drug delivery and targeted treatment across blood-brain barrier: perspectives on tracking and neuroimaging. Part Fibre Toxicol 7:3. https://doi.org/10.1186/1743-8977-7-3

Bhunia S, Vangala V, Bhattacharya D et al (2017) Large amino acid transporter 1 selective liposomes of 1-DOPA functionalized amphiphile for combating glioblastoma. Mol Pharm 14:38343847. https://doi.org/10.1021/acs.molpharmaceut.7b00569

Blasi P, Giovagnoli S, Schoubben A et al (2007) Solid lipid nanoparticles for targeted brain drug delivery. Adv Drug Deliv Rev 59:454-477. https://doi.org/10.1016/j.addr.2007.04.011

Bredlau AL, Motamarry A, Chen C et al (2018) Localized delivery of therapeutic doxorubicin dose across the canine blood-brain barrier with hyperthermia and temperature sensitive liposomes. Drug Deliv 25:973-984. https://doi.org/10.1080/10717544.2018. 1461280

Calzolari A, Larocca LM, Deaglio S et al (2010) Transferrin receptor 2 is frequently and highly expressed in glioblastomas. Transl Oncol 3:123-134. https://doi.org/10.1593/tlo.09274

Carpentier AF, Meng Y (2006) Recent advances in immunotherapy for human glioma. Curr Opin Oncol 18:631-636. https://doi.org/10. 1097/01.cco.0000245321.34658.f4

Castano AP, Demidova TN, Hamblin MR (2005) Mechanisms in photodynamic therapy: part two-cellular signaling, cell metabolism and modes of cell death. Photodiagnosis Photodyn Ther 2:1-23. https://doi.org/10.1016/S1572-1000(05)00030-X

Chamberlain MC (2010) Temozolomide: therapeutic limitations in the treatment of adult high-grade gliomas. Expert Rev Neurother 10:1537-1544. https://doi.org/10.1586/ern.10.32

Chames P, Regenmortel MV, Weiss E, Baty D (2009) Therapeutic antibodies: successes, limitations and hopes for the future. Br J Pharmacol 157:220-233. https://doi.org/10.1111/j.1476-5381. 2009.00190.x

Chauhan I, Yasir M, Verma M, Singh AP (2020) Nanostructured lipid carriers: a groundbreaking approach for transdermal drug delivery. Adv Pharm Bull 10:150-165. https://doi.org/10.34172/apb. 2020.021

Chen J, Li Y, Yu T-S et al (2012) A restricted cell population propagates glioblastoma growth after chemotherapy. Nature 488:522526. https://doi.org/10.1038/nature11287

Chen C, Duan Z, Yuan Y et al (2017) Peptide-22 and cyclic RGD functionalized liposomes for glioma targeting drug delivery overcoming BBB and BBTB. ACS Appl Mater Interfaces 9:5864-5873. https://doi.org/10.1021/acsami.6b15831

Costantini P, Jacotot E, Decaudin D, Kroemer G (2000) Mitochondrion as a novel target of anticancer chemotherapy. J Natl Cancer Inst 92:1042-1053. https://doi.org/10.1093/jnci/92.13.1042

Cui Y, Xu Q, Chow PK-H et al (2013) Transferrin-conjugated magnetic silica PLGA nanoparticles loaded with doxorubicin and paclitaxel for brain glioma treatment. Biomaterials 34:8511-8520. https://doi.org/10.1016/j.biomaterials.2013.07.075

Davis ME, Chen ZG, Shin DM (2008) Nanoparticle therapeutics: an emerging treatment modality for cancer. Nat Rev Drug Discov 7:771-782. https://doi.org/10.1038/nrd2614

DeAngelis LM (2001) Brain tumors. N Engl J Med 344:114-123. https://doi.org/10.1056/NEJM200101113440207

Deshane J, Garner CC, Sontheimer H (2003) Chlorotoxin inhibits glioma cell invasion via matrix metalloproteinase-2. J Biol Chem 278:4135-4144. https://doi.org/10.1074/jbc.M205662200

Din FU, Aman W, Ullah I, et al (2017) Effective use of nanocarriers as drug delivery systems for the treatment of selected tumors. Int $\mathbf{J}$ Nanomed 12:7291-7309. https://doi.org/10.2147/IJN.S146315
Dolmans DEJGJ, Fukumura D, Jain RK (2003) Photodynamic therapy for cancer. Nat Rev Cancer 3:380-387. https://doi.org/10.1038/ nrc1071

Ds M (2015) Regulation of ABC transporters at the blood-brain barrier. Clin Pharmacol Ther 97:395-403. https://doi.org/10.1002/cpt.64

Elsana H, Olusanya TOB, Carr-wilkinson J et al (2019) Evaluation of novel cationic gene based liposomes with cyclodextrin prepared by thin film hydration and microfluidic systems. Sci Rep 9:15120. https://doi.org/10.1038/s41598-019-51065-4

Eramo A, Ricci-Vitiani L, Zeuner A et al (2006) Chemotherapy resistance of glioblastoma stem cells. Cell Death Differ 13:1238-1241. https://doi.org/10.1038/sj.cdd.4401872

Erdő F, Bors LA, Farkas D et al (2018) Evaluation of intranasal delivery route of drug administration for brain targeting. Brain Res Bull 143:155-170. https://doi.org/10.1016/j.brainresbull.2018. 10.009

Filatova A, Acker T, Garvalov BK (2013) The cancer stem cell niche(s): the crosstalk between glioma stem cells and their microenvironment. Biochim Biophys Acta 1830:2496-2508. https://doi.org/10.1016/j.bbagen.2012.10.008

Fleige E, Quadir MA, Haag R (2012) Stimuli-responsive polymeric nanocarriers for the controlled transport of active compounds: concepts and applications. Adv Drug Deliv Rev 64:866-884. https://doi.org/10.1016/j.addr.2012.01.020

Folkins C, Shaked Y, Man S et al (2009) Glioma tumor stem-like cells promote tumor angiogenesis and vasculogenesis via vascular endothelial growth factor and stromal-derived factor 1 . Cancer Res 69:7243-7251. https://doi.org/10.1158/0008-5472. CAN-09-0167

Gan HK, van den Bent M, Lassman AB et al (2017) Antibody-drug conjugates in glioblastoma therapy: the right drugs to the right cells. Nat Rev Clin Oncol 14:695-707. https://doi.org/10.1038/ nrclinonc.2017.95

Gao H, Zhang S, Yang Z et al (2014) In vitro and in vivo intracellular distribution and anti-glioblastoma effects of docetaxel-loaded nanoparticles functioned with IL-13 peptide. Int J Pharm 466:8-17. https://doi.org/10.1016/j.ijpharm.2014.03.012

Gartziandia O, Herran E, Pedraz JL et al (2015) Chitosan coated nanostructured lipid carriers for brain delivery of proteins by intranasal administration. Colloids Surf B Biointerfaces 134:304-313. https://doi.org/10.1016/j.colsurfb.2015.06.054

Ghasemiyeh P, Mohammadi-Samani S (2018) Solid lipid nanoparticles and nanostructured lipid carriers as novel drug delivery systems: applications, advantages and disadvantages. Res Pharm Sci 13:288-303. https://doi.org/10.4103/1735-5362. 235156

Glas M, Koch H, Hirschmann B et al (2007) Pegylated liposomal doxorubicin in recurrent malignant glioma: analysis of a case series. OCL 72:302-307. https://doi.org/10.1159/000113052

Gopalan D, Pandey A, Udupa N, Mutalik S (2020) Receptor specific, stimuli responsive and subcellular targeted approaches for effective therapy of Alzheimer: role of surface engineered nanocarriers. J Control Release 319:183-200. https://doi.org/ 10.1016/j.jconrel.2019.12.034

Gulsun T, Izat N, Sahin S (2017) Chapter 3 - Brain and the Drug Transporters. In: Gürsoy-Özdemir Y, Bozdağ-Pehlivan S, Sekerdag E (eds) Nanotechnology methods for neurological diseases and brain tumors. Academic Press, pp 35-67

Guo J, Gao X, Su L et al (2011) Aptamer-functionalized PEG-PLGA nanoparticles for enhanced anti-glioma drug delivery. Biomaterials 32:8010-8020. https://doi.org/10.1016/j.biomaterials. 2011.07.004

Hanif S, Muhammad P, Chesworth R et al (2020) Nanomedicinebased immunotherapy for central nervous system disorders. Acta Pharmacol Sin 41:936-953. https://doi.org/10.1038/ s41401-020-0429-z 
Hayward SL, Wilson CL, Kidambi S (2016) Hyaluronic acid-conjugated liposome nanoparticles for targeted delivery to CD44 overexpressing glioblastoma cells. Oncotarget 7:34158-34171. https://doi.org/10.18632/oncotarget.8926

$\mathrm{He}$ C, Hu Y, Yin L et al (2010) Effects of particle size and surface charge on cellular uptake and biodistribution of polymeric nanoparticles. Biomaterials 31:3657-3666. https://doi.org/ 10.1016/j.biomaterials.2010.01.065

Hervé F, Ghinea N, Scherrmann J-M (2008) CNS delivery via adsorptive transcytosis. AAPS J 10:455-472. https://doi.org/ 10.1208/s12248-008-9055-2

Hjelmeland AB, Wu Q, Heddleston JM et al (2011) Acidic stress promotes a glioma stem cell phenotype. Cell Death Differ 18:829840. https://doi.org/10.1038/cdd.2010.150

Ho L, Bokharaei M, Li S-D (2018) Current update of a thermosensitive liposomes composed of DPPC and Brij78. J Drug Target 26:407-419. https://doi.org/10.1080/1061186X.2017.1419361

Hua L, Wang Z, Zhao L et al (2018) Hypoxia-responsive lipid-poly(hypoxic radiosensitized polyprodrug) nanoparticles for glioma chemo- and radiotherapy. Theranostics 8:5088-5105. https://doi. org/10.7150/thno.26225

Huang Z, Cheng L, Guryanova OA et al (2010) Cancer stem cells in glioblastoma-molecular signaling and therapeutic targeting. Protein Cell 1:638-655. https://doi.org/10.1007/s13238-010-0078-y

Huang R, Ke W, Han L et al (2011) Targeted delivery of chlorotoxinmodified DNA-loaded nanoparticles to glioma via intravenous administration. Biomaterials 32:2399-2406. https://doi.org/10. 1016/j.biomaterials.2010.11.079

Illum L (2000) Transport of drugs from the nasal cavity to the central nervous system. Eur J Pharm Sci 11:1-18. https://doi.org/10. 1016/s0928-0987(00)00087-7

Iqbal MA, Md S, Sahni JK et al (2012) Nanostructured lipid carriers system: recent advances in drug delivery. J Drug Target 20:813830. https://doi.org/10.3109/1061186X.2012.716845

Jhaveri A, Torchilin V (2016) Intracellular delivery of nanocarriers and targeting to subcellular organelles. Expert Opin Drug Deliv 13:49-70. https://doi.org/10.1517/17425247.2015.1086745

Jhaveri A, Deshpande P, Pattni B, Torchilin V (2018) Transferrin-targeted, resveratrol-loaded liposomes for the treatment of glioblastoma. J Control Release 277:89-101. https://doi.org/10.1016/j. jconrel.2018.03.006

Johnsen KB, Burkhart A, Melander F et al (2017) Targeting transferrin receptors at the blood-brain barrier improves the uptake of immunoliposomes and subsequent cargo transport into the brain parenchyma. Sci Rep 7:1-13. https://doi.org/10.1038/ s41598-017-11220-1

Kadari A, Pooja D, Gora RH et al (2018) Design of multifunctional peptide collaborated and docetaxel loaded lipid nanoparticles for antiglioma therapy. Eur J Pharm Biopharm 132:168-179. https:// doi.org/10.1016/j.ejpb.2018.09.012

Karanth H, Murthy RSR (2007) pH-sensitive liposomes-principle and application in cancer therapy. J Pharm Pharmacol 59:469-483. https://doi.org/10.1211/jpp.59.4.0001

Karim R, Palazzo C, Evrard B, Piel G (2016) Nanocarriers for the treatment of glioblastoma multiforme: current state-of-the-art. J Control Release 227:23-37. https://doi.org/10.1016/j.jconrel. 2016.02.026

Khames A, Khaleel MA, El-Badawy MF, El-Nezhawy AOH (2019) Natamycin solid lipid nanoparticles-sustained ocular delivery system of higher corneal penetration against deep fungal keratitis: preparation and optimization. Int J Nanomed 14:2515-2531. https://doi.org/10.2147/IJN.S190502

Khan A, Imam SS, Aqil M et al (2016) Brain targeting of temozolomide via the intranasal route using lipid-based nanoparticles: brain pharmacokinetic and scintigraphic analyses. Mol Pharm
13:3773-3782. https://doi.org/10.1021/acs.molpharmaceut. $6 \mathrm{~b} 00586$

Khan AR, Yang X, Fu M, Zhai G (2018) Recent progress of drug nanoformulations targeting to brain. J Control Release 291:37-64. https://doi.org/10.1016/j.jconrel.2018.10.004

Kievit FM, Veiseh O, Fang C et al (2010) Chlorotoxin labeled magnetic nanovectors for targeted gene delivery to glioma. ACS Nano 4:4587-4594. https://doi.org/10.1021/nn1008512

Kim JS, Shin DH, Kim J-S (2018) Dual-targeting immunoliposomes using angiopep-2 and CD133 antibody for glioblastoma stem cells. J Control Release 269:245-257. https://doi.org/10.1016/j. jconrel.2017.11.026

Kono K (2001) Thermosensitive polymer-modified liposomes. Adv Drug Deliv Rev 53:307-319. https://doi.org/10.1016/s0169409x(01)00204-6

Koukourakis MI, Koukouraki S, Fezoulidis I et al (2000) High intratumoural accumulation of stealth liposomal doxorubicin (Caelyx) in glioblastomas and in metastatic brain tumours. Br J Cancer 83:1281-1286. https://doi.org/10.1054/bjoc.2000.1459

Krammer B (2001) Vascular effects of photodynamic therapy. Anticancer Res 21:4271-4277

Küçüktürkmen B, Devrim B, Saka OM et al (2017) Co-delivery of pemetrexed and miR-21 antisense oligonucleotide by lipidpolymer hybrid nanoparticles and effects on glioblastoma cells. Drug Dev Ind Pharm 43:12-21. https://doi.org/10.1080/03639 045.2016.1200069

Kumar H, Mishra G, Sharma AK et al (2017) Intranasal drug delivery: a non-invasive approach for the better delivery of neurotherapeutics. Pharm Nanotechnol 5:203-214. https://doi.org/10.2174/ 2211738505666170515113936

Lambert DM (2000) Rationale and applications of lipids as prodrug carriers. Eur J Pharm Sci 11(Suppl 2):15-27. https://doi.org/10. 1016/s0928-0987(00)00161-5

Lee Y, Thompson DH (2017) Stimuli-responsive liposomes for drug delivery. Wiley Interdiscip Rev Nanomed Nanobiotechnol. https://doi.org/10.1002/wnan.1450

Li W-T (2013) Nanoparticles for photodynamic therapy. In: Handbook of biophotonics. American Cancer Society, Atlanta, pp 321-336

Li Y, Sahi J (2016) The role of drug transporters at the blood brain barrier. Eur Pharm Rev 21:15-19

Li M, Shi K, Tang X et al (2018) pH-sensitive folic acid and dNP2 peptide dual-modified liposome for enhanced targeted chemotherapy of glioma. Eur J Pharm Sci 124:240-248. https://doi. org/10.1016/j.ejps.2018.07.055

Li A, Zhao J, Fu J et al (2021) Recent advances of biomimetic nanosystems in the diagnosis and treatment of tumor. Asian J Pharm Sci 16:161-174. https://doi.org/10.1016/j.ajps.2019.08.001

Liao W, Fan S, Zheng Y et al (2019) Recent advances on glioblastoma multiforme and nano-drug carriers: a review. Curr Med Chem 26:5862-5874. https://doi.org/10.2174/09298673256661805141 13136

Licha K, Riefke B, Ntziachristos V et al (2000) Hydrophilic cyanine dyes as contrast agents for near-infrared tumor imaging: synthesis, photophysical properties and spectroscopic in vivo characterization. Photochem Photobiol 72:392-398. https://doi.org/ $10.1562 / 0031$

Liu Y, Lu W (2012) Recent advances in brain tumor-targeted nano-drug delivery systems. Expert Opin Drug Deliv 9:671-686. https:// doi.org/10.1517/17425247.2012.682726

Liu G, Shen H, Mao J et al (2013) Transferrin modified graphene oxide for glioma-targeted drug delivery: in vitro and in vivo evaluations. ACS Appl Mater Interfaces 5:6909-6914. https://doi.org/ 10.1021/am402128s

Liu C, Liu X-N, Wang G-L et al (2017a) A dual-mediated liposomal drug delivery system targeting the brain: rational construction, integrity evaluation across the blood-brain barrier, and the 
transporting mechanism to glioma cells. Int J Nanomed 12:24072425. https://doi.org/10.2147/IJN.S131367

Liu H, Xie Y, Zhang Y et al (2017b) Development of a hypoxia-triggered and hypoxic radiosensitized liposome as a doxorubicin carrier to promote synergetic chemo-/radio-therapy for glioma. Biomaterials 121:130-143. https://doi.org/10.1016/j.biomateria 1s.2017.01.001

Liu H-M, Zhang Y-F, Xie Y-D et al (2017c) Hypoxia-responsive ionizable liposome delivery siRNA for glioma therapy. Int J Nanomed 12:1065-1083. https://doi.org/10.2147/IJN.S125286

Lombardo D, Kiselev MA, Caccamo MT (2019) Smart nanoparticles for drug delivery application: development of versatile nanocarrier platforms in biotechnology and nanomedicine. J Nanomater 26:1687-4110. https://doi.org/10.1155/2019/3702518

Lonez C, Vandenbranden M, Ruysschaert J-M (2008) Cationic liposomal lipids: from gene carriers to cell signaling. Prog Lipid Res 47:340-347. https://doi.org/10.1016/j.plipres.2008.03.002

Lou J, Zhang L, Zheng G (2019) Advancing cancer immunotherapies with nanotechnology. Adv Ther 2:1800128. https://doi.org/10. 1002/adtp.201800128

Lu C-T, Zhao Y-Z, Wong HL et al (2014) Current approaches to enhance CNS delivery of drugs across the brain barriers. Int J Nanomed 9:2241-2257. https://doi.org/10.2147/IJN.S61288

Lucky SS, Soo KC, Zhang Y (2015) Nanoparticles in photodynamic therapy. Chem Rev 115:1990-2042. https://doi.org/10.1021/ cr5004198

Luo D, Li N, Carter KA et al (2016) Rapid light-triggered drug release in liposomes containing small amounts of unsaturated and porphyrin-phospholipids. Small 12:3039-3047. https://doi.org/10. 1002/smll.201503966

Madane RG, Mahajan HS (2016) Curcumin-loaded nanostructured lipid carriers (NLCs) for nasal administration: design, characterization, and in vivo study. Drug Deliv 23:1326-1334. https:// doi.org/10.3109/10717544.2014.975382

Mahmud H, Kasai T, Khayrani AC et al (2018) Targeting glioblastoma cells expressing CD44 with liposomes encapsulating doxorubicin and displaying chlorotoxin-IgG Fc fusion protein. Int J Mol Sci 19:659. https://doi.org/10.3390/ijms19030659

Mansor NI, Nordin N, Mohamed F, Ling KH, Rosli R, Hassan Z (2019) Crossing the blood-brain barrier: a review on drug delivery strategies for treatment of the central nervous system diseases. Curr Drug Deliv 16:698-711. https://doi.org/10.2174/1567201816 666190828153017

Marino A, Camponvo A, Degl'Innocenti A et al (2019) Multifunctional temozolomide-loaded lipid superparamagnetic nanovectors: dual targeting and disintegration of glioblastoma spheroids by synergic chemotherapy and hyperthermia treatment. Nanoscale 11:21227-21248. https://doi.org/10.1039/C9NR07976A

Mason WP (2015) Blood-brain barrier-associated efflux transporters: a significant but underappreciated obstacle to drug development in glioblastoma. Neuro Oncol 17:1181-1182. https://doi.org/10. 1093/neuonc/nov122

Mishra S, Sharma S, Javed MN et al (2019) Bioinspired nanocomposites: applications in disease diagnosis and treatment. Pharm Nanotechnol 7:206-219. https://doi.org/10.2174/2211738507 666190425121509

Momoi T (2004) Caspases involved in ER stress-mediated cell death. J Chem Neuroanat 28:101-105. https://doi.org/10.1016/j.jchem neu.2004.05.008

Monaco I, Camorani S, Colecchia D et al (2017) Aptamer functionalization of nanosystems for glioblastoma targeting through the blood-brain barrier. J Med Chem 60:4510-4516. https://doi.org/ 10.1021/acs.jmedchem.7b00527

Mukherjee A, Waters AK, Kalyan P et al (2019) Lipid-polymer hybrid nanoparticles as a next-generation drug delivery platform: state of the art, emerging technologies, and perspectives. Int $\mathbf{J}$ Nanomed 14:1937-1952. https://doi.org/10.2147/IJN.S198353

Müller RH, Mäder K, Gohla S (2000) Solid lipid nanoparticles (SLN) for controlled drug delivery - a review of the state of the art. Eur J Pharm Biopharm 50:161-177. https://doi.org/10.1016/s09396411(00)00087-4

Mura S, Nicolas J, Couvreur P (2013) Stimuli-responsive nanocarriers for drug delivery. Nat Mater 12:991-1003. https://doi.org/ 10.1038/nmat3776

Nikanjam M, Gibbs AR, Hunt CA et al (2007) Synthetic nano-LDL with paclitaxel oleate as a targeted drug delivery vehicle for glioblastoma multiforme. J Control Release 124:163-171. https://doi. org/10.1016/j.jconrel.2007.09.007

Niu X, Chen J, Gao J (2019) Nanocarriers as a powerful vehicle to overcome blood-brain barrier in treating neurodegenerative diseases: Focus on recent advances. Asian J Pharm Sci 14:480-496. https://doi.org/10.1016/j.ajps.2018.09.005

Norouzi M (2020) Gold nanoparticles in glioma theranostics. Pharmacol Res 156:104753. https://doi.org/10.1016/j.phrs.2020.104753

Norouzi M, Yathindranath V, Thliveris JA, Miller DW (2020) Salinomycin-loaded iron oxide nanoparticles for glioblastoma therapy. Nanomaterials (basel). https://doi.org/10.3390/nano10030477

Ordys BB, Launay S, Deighton RF et al (2010) The role of mitochondria in glioma pathophysiology. Mol Neurobiol 42:64-75. https:// doi.org/10.1007/s12035-010-8133-5

Pacheco-Torres J, Mukherjee N, Walko M et al (2015) Image guided drug release from $\mathrm{pH}$-sensitive Ion channel-functionalized stealth liposomes into an in vivo glioblastoma model. Nanomedicine 11:1345-1354. https://doi.org/10.1016/j.nano.2015.03.014

Pardeike J, Hommoss A, Müller RH (2009) Lipid nanoparticles (SLN, NLC) in cosmetic and pharmaceutical dermal products. Int $\mathbf{J}$ Pharm 366:170-184. https://doi.org/10.1016/j.ijpharm.2008.10. 003

Pardeshi C, Rajput P, Belgamwar V, Tekade A, Patil G, Chaudhary K, Sonje A (2012) Solid lipid based nanocarriers: an overview. Acta Pharm 62:433-472. https://doi.org/10.2478/v10007-012-0040-z

Parodi A, Corbo C, Cevenini A et al (2015) Enabling cytoplasmic delivery and organelle targeting by surface modification of nanocarriers. Nanomedicine 10:1923. https://doi.org/10.2217/nnm. 15.39

Perry JR, Laperriere N, O'Callaghan CJ et al (2017) Short-course radiation plus temozolomide in elderly patients with glioblastoma. N Engl J Med 376:1027-1037. https://doi.org/10.1056/ NEJMoa1611977

Pinton L, Magri S, Masetto E et al (2020) Targeting of immunosuppressive myeloid cells from glioblastoma patients by modulation of size and surface charge of lipid nanocapsules. J Nanobiotechnology 18:31. https://doi.org/10.1186/s12951-020-00589-3

Poonia N, Kharb R, Lather V, Pandita D (2016) Nanostructured lipid carriers: versatile oral delivery vehicle. Future Sci. https://doi. org/10.4155/fsoa-2016-0030

Pottoo FH, Javed MN, Rahman JU et al (2020) Targeted delivery of miRNA based therapeuticals in the clinical management of glioblastoma multiforme. Semin Cancer Biol. https://doi.org/ 10.1016/j.semcancer.2020.04.001

Prabhu S, Goda JS, Mutalik S, Mohanty BS, Chaudhari P, Rai S, Udupa N, Rao BSS et al (2017) A polymeric temozolomide nanocomposite against orthotopic glioblastoma xenograft: tumor-specific homing directed by nestin. Nanoscale 30:10919-10932. https:// doi.org/10.1039/C7NR00305F

Puri A, Loomis K, Smith B et al (2009) Lipid-based nanoparticles as pharmaceutical drug carriers: from concepts to clinic. Crit Rev Ther Drug Carrier Syst 26:523-580

Qin Y, Fan W, Chen $\mathrm{H}$ et al (2010) In vitro and in vivo investigation of glucose-mediated brain-targeting liposomes. J Drug Target 18:536-549. https://doi.org/10.3109/10611861003587235 
Radwan AA, Alanazi FK (2014) Targeting cancer using cholesterol conjugates. Saudi Pharm J 22:3-16. https://doi.org/10.1016/j. jsps.2013.01.003

Rajendran L, Knölker H-J, Simons K (2010) Subcellular targeting strategies for drug design and delivery. Nat Rev Drug Discov 9:29-42. https://doi.org/10.1038/nrd2897

Raucher D (2019) Tumor targeting peptides: novel therapeutic strategies in glioblastoma. Curr Opin Pharmacol 47:14-19. https://doi. org/10.1016/j.coph.2019.01.006

Rey S, Schito L, Koritzinsky M, Wouters BG (2017) Molecular targeting of hypoxia in radiotherapy. Adv Drug Deliv Rev 109:45-62. https://doi.org/10.1016/j.addr.2016.10.002

Riaz MK, Riaz MA, Zhang X et al (2018) Surface functionalization and targeting strategies of liposomes in solid tumor therapy: a review. Int J Mol Sci 19:195. https://doi.org/10.3390/ijms19010195

Rip J, Schenk GJ, de Boer A (2009) Differential receptor-mediated drug targeting to the diseased brain. Expert Opin Drug Deliv 6:227-237. https://doi.org/10.1517/17425240902806383

Sahebjam S, Sharabi A, Lim M et al (2017) Immunotherapy and radiation in glioblastoma. J Neurooncol 134:531-539. https://doi.org/ 10.1007/s11060-017-2413-0

Sanna V, Pala N, Sechi M (2014) Targeted therapy using nanotechnology: focus on cancer. Int J Nanomed 9:467-483. https://doi.org/ 10.2147/IJN.S36654

Sarangi MK, Padhi S (2016) Solid lipid nanoparticles-a review. J Crit Rev 3:5-12

Saxena V, Sadoqi M, Shao J (2003) Degradation kinetics of indocyanine green in aqueous solution. J Pharm Sci 92:2090-2097. https://doi.org/10.1002/jps.10470

Sekerdag E, Lüle S, Bozdağ Pehlivan S et al (2017) A potential noninvasive glioblastoma treatment: nose-to-brain delivery of farnesylthiosalicylic acid incorporated hybrid nanoparticles. J Control Release 261:187-198. https://doi.org/10.1016/j.jconrel. 2017.06.032

Selvamuthukumar S, Velmurugan R (2012) Nanostructured lipid carriers: a potential drug carrier for cancer chemotherapy. Lipids Health Dis 11:159. https://doi.org/10.1186/1476-511X-11-159

Sercombe L, Veerati T, Moheimani F et al (2015) Advances and challenges of liposome assisted drug delivery. Front Pharmacol. https://doi.org/10.3389/fphar.2015.00286

Shafirstein G, Battoo A, Harris K et al (2016) Photodynamic therapy of non-small cell lung cancer. Narrative review and future directions. Ann Am Thorac Soc 13:265-275. https://doi.org/10.1513/ AnnalsATS.201509-650FR

Shah R, Eldridge D, Palombo E, Harding I (2015) Lipid nanoparticles: production, characterization and stability. Springer, Cham

Shein SA, Kuznetsov II, Abakumova TO et al (2016) VEGF- and VEGFR2-targeted liposomes for cisplatin delivery to glioma cells. Mol Pharm 13:3712-3723. https://doi.org/10.1021/acs. molpharmaceut.6b00519

Shen S, Khazaeli MB, Gillespie GY, Alvarez VL (2005) Radiation dosimetry of 131 I-chlorotoxin for targeted radiotherapy in glioma-bearing mice. J Neurooncol 71:113-119. https://doi.org/10. 1007/s11060-004-0890-4

Shi K, Zhou J, Zhang Q et al (2015) Arginine-glycine-aspartic acidmodified lipid-polymer hybrid nanoparticles for docetaxel delivery in glioblastoma multiforme.J. Biomed Nanotechnol 11:382391. https://doi.org/10.1166/jbn.2015.1965

Shi D, Mi G, Shen Y, Webster TJ (2019) Glioma-targeted dual functionalized thermosensitive Ferri-liposomes for drug delivery through an in vitro blood-brain barrier. Nanoscale 11:1505715071. https://doi.org/10.1039/C9NR03931G

Shibata S, Shinozaki N, Suganami A et al (2019) Photo-immune therapy with liposomally formulated phospholipid-conjugated indocyanine green induces specific antitumor responses with heat shock protein-70 expression in a glioblastoma model. Oncotarget 10:175-183. https://doi.org/10.18632/oncotarget.26544

Shin DH, Lee S-J, Kim JS et al (2015) Synergistic effect of immunoliposomal gemcitabine and bevacizumab in glioblastoma stem cell-targeted therapy. J Biomed Nanotechnol 11:1989-2002. https://doi.org/10.1166/jbn.2015.2146

Shu JY, Panganiban B, Xu T (2013) Peptide-polymer conjugates: from fundamental science to application. Annu Rev Phys Chem 64:631-657. https://doi.org/10.1146/annurev-physc hem-040412-110108

Shukla T, Upmanyu N, Prakash Pandey S, Gosh D (2018) Lipid nanocarriers. In: Grumezescu AM (ed) Lipid nanocarriers for drug targeting. William Andrew Publishing, Norwich, pp 1-47

Silvestre DC, Maccioni HJF, Caputto BL (2009) Content of endoplasmic reticulum and Golgi complex membranes positively correlates with the proliferative status of brain cells. J Neurosci Res 87:857-865. https://doi.org/10.1002/jnr.21915

Soeda A, Park M, Lee D et al (2009) Hypoxia promotes expansion of the CD133-positive glioma stem cells through activation of HIF-1 $\alpha$. Oncogene 28:3949-3959. https://doi.org/10.1038/onc. 2009.252

Song S, Mao G, Du J, Zhu X (2016) Novel RGD containing, temozolomide-loading nanostructured lipid carriers for glioblastoma multiforme chemotherapy. Drug Deliv 23:1404-1408. https:// doi.org/10.3109/10717544.2015.1064186

Soroceanu L, Gillespie Y, Khazaeli MB, Sontheimer H (1998) Use of chlorotoxin for targeting of primary brain tumors. Cancer Res 58:4871-4879

Sriraman SK, Salzano G, Torchilin V (2017) Drug delivery to the central nervous system. In: Caplan LR, Biller J, Leary MC et al (eds) Primer on cerebrovascular diseases, 2nd edn. Academic Press, San Diego, pp 198-201

Stupp R, Tonn J-C, Brada M, Pentheroudakis G (2010) High-grade malignant glioma: ESMO clinical practice guidelines for diagnosis, treatment and follow-up. Ann Oncol 21:190-193. https:// doi.org/10.1093/annonc/mdq187

Su Z, Xing L, Chen Y et al (2014) Lactoferrin-modified poly(ethylene glycol)-grafted BSA nanoparticles as a dual-targeting carrier for treating brain gliomas. Mol Pharm 11:1823-1834. https://doi. org/10.1021/mp500238m

Świętek M, Panchuk R, Skorokhyd N et al (2020) Magnetic temperature-sensitive solid-lipid particles for targeting and killing tumor cells. Front Chem. https://doi.org/10.3389/fchem.2020.00205

Tapeinos C, Battaglini M, Ciofani G (2017) Advances in the design of solid lipid nanoparticles and nanostructured lipid carriers for targeting brain diseases. J Control Release 264:306-332. https:// doi.org/10.1016/j.jconrel.2017.08.033

Üner M, Yener G (2007) Importance of solid lipid nanoparticles (SLN) in various administration routes and future perspectives. Int $\mathbf{J}$ Nanomed 2:289-300

van Tellingen O, Yetkin-Arik B, de Gooijer MC et al (2015) Overcoming the blood-brain tumor barrier for effective glioblastoma treatment. Drug Resist Updat 19:1-12. https://doi.org/10.1016/j. drup.2015.02.002

van Tellingen O, de Gooijer MC, Buil LCM et al (2018) P04.91 ABCdrug efflux transporters at the blood-brain barrier limit the efficacy of treatment against primary and secondary brain tumors. Neuro Oncol 20:iii301. https://doi.org/10.1093/neuonc/noy139. 325

Voth B, Nagasawa DT, Pelargos PE et al (2015) Transferrin receptors and glioblastoma multiforme: current findings and potential for treatment. J Clin Neurosci 22:1071-1076. https://doi.org/10. 1016/j.jocn.2015.02.002

Wang G, Wang JJ, Yang GY et al (2012) Effects of quercetin nanoliposomes on C6 glioma cells through induction of type III 
programmed cell death. Int J Nanomed 7:271-280. https://doi. org/10.2147/IJN.S26935

Wang S, Meng Y, Li C et al (2015) Receptor-mediated drug delivery systems targeting to glioma. Nanomaterials (basel). https://doi. org/10.3390/nano6010003

Wang K, Kievit FM, Zhang M (2016) Nanoparticles for cancer gene therapy: recent advances, challenges, and strategies. Pharmacol Res 114:56-66. https://doi.org/10.1016/j.phrs.2016.10.016

Warren KE (2018) Beyond the blood: brain barrier: the importance of central nervous system (CNS) pharmacokinetics for the treatment of CNS tumors, including diffuse intrinsic pontine glioma. Front Oncol. https://doi.org/10.3389/fonc.2018.00239

Xiang Y, Liang L, Wang X et al (2011) Chloride channel-mediated brain glioma targeting of chlorotoxin-modified doxorubicineloaded liposomes. J Control Release 152:402-410. https://doi. org/10.1016/j.jconrel.2011.03.014

Xie Y, Han Y, Zhang X et al (2021) Application of new radiosensitizer based on nano-biotechnology in the treatment of glioma. Front Oncol. https://doi.org/10.3389/fonc.2021.633827

Xu H, Han Y, Zhao G et al (2020) Hypoxia-responsive lipid-polymer nanoparticle-combined imaging-guided surgery and multitherapy strategies for glioma. ACS Appl Mater Interfaces 12:5231952328. https://doi.org/10.1021/acsami.0c12971

Yang Z-Z, Gao W, Liu Y-J et al (2017) Delivering siRNA and chemotherapeutic molecules across BBB and BTB for intracranial glioblastoma therapy. Mol Pharm 14:1012-1022. https://doi.org/10. 1021/acs.molpharmaceut.6b00819

Yang Q, Zhou Y, Chen J et al (2021) gene therapy for drug-resistant glioblastoma via lipid-polymer hybrid nanoparticles combined with focused ultrasound. Int J Nanomed 16:185-199. https://doi. org/10.2147/IJN.S286221

Yatvin MB, Weinstein JN, Dennis WH, Blumenthal R (1978) Design of liposomes for enhanced local release of drugs by hyperthermia. Science 202:1290-1293. https://doi.org/10.1126/science.364652

Yingchoncharoen P, Kalinowski DS, Richardson DR (2016) Lipidbased drug delivery systems in cancer therapy: what is available and what is yet to come. Pharmacol Rev 68:701-787. https://doi. org/10.1124/pr.115.012070

Yuba E (2020) Development of functional liposomes by modification of stimuli-responsive materials and their biomedical applications.
J Mater Chem B 8:1093-1107. https://doi.org/10.1039/C9TB0 $2470 \mathrm{~K}$

Zeromski J (2002) Significance of tumor-cell receptors in human cancer. Arch Immunol Ther Exp (warsz) 50:105-110

Zhang L, Gu FX, Chan JM et al (2008) Nanoparticles in medicine: therapeutic applications and developments. Clin Pharmacol Ther 83:761-769. https://doi.org/10.1038/sj.clpt.6100400

Zhang F, Stephan SB, Ene CI et al (2018) Nanoparticles that reshape the tumor milieu create a therapeutic window for effective $\mathrm{T}$ cell therapy in solid malignancies. Cancer Res 78:3718-3730. https:// doi.org/10.1158/0008-5472.CAN-18-0306

Zhao W-Y, Zhang C-X, Liu L et al (2016a) Construction of functional targeting daunorubicin liposomes used for eliminating brain glioma and glioma stem cells. J Biomed Nanotechnol 12:14041420. https://doi.org/10.1166/jbn.2016.2266

Zhao Y, Ren W, Zhong T et al (2016b) Tumor-specific pH-responsive peptide-modified $\mathrm{pH}$-sensitive liposomes containing doxorubicin for enhancing glioma targeting and anti-tumor activity. J Control Release 222:56-66. https://doi.org/10.1016/j.jconrel.2015.12.006

Zhao J, Liu P, Ma J et al (2019) Enhancement of radiosensitization by silver nanoparticles functionalized with polyethylene glycol and aptamer as1411 for glioma irradiation therapy. Int J Nanomed 14:9483-9496. https://doi.org/10.2147/IJN.S224160

Zhao M, van Straten D, Broekman MLD et al (2020) Nanocarrierbased drug combination therapy for glioblastoma. Theranostics 10:1355-1372. https://doi.org/10.7150/thno.38147

Zheng C, Ma C, Bai E et al (2015) Transferrin and cell-penetrating peptide dual-functioned liposome for targeted drug delivery to glioma. Int J Clin Exp Med 8:1658-1668

Zhu Y, Liao L (2015) Applications of nanoparticles for anticancer drug delivery: a review. J Nanosci Nanotechnol 15:4753-4773. https:// doi.org/10.1166/jnn.2015.10298

Zong Z, Hua L, Wang Z et al (2019) Self-assembled angiopep-2 modified lipid-poly (hypoxic radiosensitized polyprodrug) nanoparticles delivery TMZ for glioma synergistic TMZ and RT therapy. Drug Deliv 26:34-44. https://doi.org/10.1080/10717544.2018. 1534897

Publisher's Note Springer Nature remains neutral with regard to jurisdictional claims in published maps and institutional affiliations. 